\title{
TOPOLOGICAL COHOCHSCHILD HOMOLOGY AND THE HOMOLOGY OF FREE LOOP SPACES
}

\author{
ANNA MARIE BOHMANN, TEENA GERHARDT, AND BROOKE SHIPLEY
}

\begin{abstract}
We study the homology of free loop spaces via techniques arising from the theory of topological coHochschild homology (coTHH). Topological coHochschild homology is a topological analogue of the classical theory of coHochschild homology for coalgebras. We produce new spectrum-level structure on coTHH of suspension spectra as well as new algebraic structure in the coBökstedt spectral sequence for computing coTHH. These new techniques allow us to compute the homology of free loop spaces in several new cases, extending known calculations.
\end{abstract}

\section{INTRODUCTION}

Given a space $X$, the free loop space $\mathcal{L} X$ is the space of all maps from the circle $S^{1}$ into $X$. The (co)homology of free loop spaces has been an active area of research for many decades. Interest in this area stems from a number of important applications of free loop spaces to topology and mathematical physics. For example, a classical theorem of Gromoll and Meyer [18] ties the homology of free loop spaces to the enumeration of closed geodesics on manifolds. The homology of free loop spaces is also the main object of study in the field of string topology [11, 13].

One of the primary strategies for understanding the (co)homology of free loop spaces is to relate it to Hochschild homology [17, 10, 30]. In this paper, we use the dual construction, coHochschild homology, and the emerging theory of topological coHochschild homology to develop new tools for calculating the homology of free loop spaces. We also describe new algebraic structure on the homology of free loop spaces.

Recall that topological Hochschild homology (THH) is an analogue for ring spectra of the classical theory of Hochschild homology for algebras. Similarly, the classical theory of coHochschild homology for coalgebras [14, 9] has a topological analogue for coalgebras in spectra, called topological coHochschild homology (coTHH). The foundations of coTHH have been developed in work of Hess and Shipley [20] as well as work of Bohmann, Gerhardt, Høgenhaven, Shipley, and Ziegenhagen [4].

Some important examples of coalgebras in spectra are given by suspension spectra of spaces. Indeed, for a space $X$, the diagonal map on $X$ induces a comultiplication

$$
\triangle: \Sigma_{+}^{\infty} X \rightarrow \Sigma_{+}^{\infty} X \wedge \Sigma_{+}^{\infty} X
$$

Date: December 15, 2021.

2020 Mathematics Subject Classification. Primary:55P35, 16T15; Secondary: 55P43, 13D03, 16T05, 55T99.

Key words and phrases. Topological Hochschild homology, coalgebra, free loop spaces. 
which makes $\Sigma_{+}^{\infty} X$ into a coalgebra. For $X$ a simply connected space, $\operatorname{coTHH}\left(\Sigma_{+}^{\infty} X\right)$ can be identified with the suspension spectrum of the free loop space on $X$,

$$
\operatorname{coTHH}\left(\Sigma_{+}^{\infty} X\right) \simeq \Sigma_{+}^{\infty} \mathcal{L} X .
$$

Thus tools to study topological coHochschild homology in particular yield information about free loop spaces.

For topological Hochschild homology, one of the primary computational tools is the Bökstedt spectral sequence, which relates the homology of THH with the algebraic theory of Hochschild homology. For a field $k$ and a ring spectrum $R$, this has the form

$$
E_{*, *}^{2}=\mathrm{HH}_{*}\left(H_{*}(R ; k)\right) \Rightarrow H_{*}(\mathrm{THH}(R) ; k) .
$$

In prior work, the authors and collaborators constructed an analogous coBökstedt spectral sequence computing the homology of coTHH [4]. For a field $k$ and a coalgebra spectrum $C$, this spectral sequence has $E_{2}$-term

$$
E_{2}^{s, t}=\operatorname{coHH}_{s, t}\left(H_{*}(C ; k)\right)
$$

and abuts to $H_{t-s}(\operatorname{coTHH}(C) ; k)$. Here coHH is the classical coHochschild homology for coalgebras of Doi [14]. In the case of suspension spectra of simply connected spaces, the coBökstedt spectral sequence converges if a Mittag-Leffler condition is satisfied, and gives computational tools to study the homology of free loop spaces:

$$
H_{*}\left(\operatorname{coTHH}\left(\Sigma_{+}^{\infty} X\right) ; k\right) \cong H_{*}(\mathcal{L} X ; k) .
$$

The current paper studies the algebraic structure in the coBökstedt spectral sequence, with an eye towards applications to the homology of free loop spaces. Work of Angeltveit and Rognes [2] shows that under appropriate flatness conditions, the Bökstedt spectral sequence computing $H_{*}(\mathrm{THH}(R) ; k)$ is a spectral sequence of $H_{*}(R ; k)$-Hopf algebras. One might then expect a dual algebraic structure in the coBökstedt spectral sequence. This is more subtle than it seems, however, because such an algebraic structure has a multiplication and comultiplication over the coalgebra $H_{*}(C ; k)$, rather than over a ring. In this paper we define the appropriate Hopf algebra-like structure over a coalgebra $D$, which we call a $\square_{D}$-Hopf algebra. A $\square_{D}$-Hopf algebra $H$ is a $D$-bicomodule with appropriately compatible multiplication and comultiplication maps

$$
\mu: H \square_{D} H \rightarrow H \quad \text { and } \quad \triangle: H \rightarrow H \square_{D} H,
$$

where $\square_{D}$ denotes the cotensor product over the coalgebra $D$.

These new constructions allow us to describe new algebraic structure on the homology of free loop spaces.

Theorem 1.1. For $X$ a simply connected space and $k$ a field, if $H_{*}(\mathcal{L} X ; k)$ is coflat as a comodule over $H_{*}(X ; k)$, then $H_{*}(\mathcal{L} X ; k)$ is a $\square_{H_{*}(X ; k)}$-Hopf algebra.

We obtain this algebraic result as the consequence of spectrum-level structure arising in the $\infty$-category of coalgebra spectra over the suspension spectrum $\Sigma_{+}^{\infty} X$, which is discussed in Proposition 5.6.

We then prove the following result capturing the algebraic structure in the coBökstedt spectral sequence: 
Theorem 1.2. Let $C$ be a connected cocommutative coalgebra spectrum. The

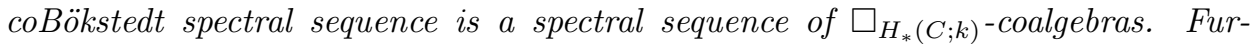
ther, if for each $r \geq 2, E_{r}^{*, *}(C)$ is coflat over $H_{*}(C ; k)$, then the coBökstedt spectral sequence is a spectral sequence of $\square_{H_{*}(C ; k)}$-Hopf algebras.

This algebraic structure in the coBökstedt spectral sequence facilitates new computations of $H_{*}(\operatorname{coTHH}(C) ; k)$, and in particular of the homology of free loop spaces $H_{*}(\mathcal{L} X ; k)$.

In this work, we illustrate the power of this structure on the spectral sequence by considering the homology of free loop spaces on simply connected spaces $X$ with certain cohomology rings. We consider in detail the homology of $\mathcal{L} X$ when $X$ is a simply connected space with exterior cohomology. The (co)homology of $\mathcal{L} X$ in such cases has been considered, for instance, in [25], [23], and [24]. Our approach yields new results.

Theorem 1.3. Let $k$ be a field of characteristic $p$ and let $X$ be a simply connected space whose cohomology is exterior on a finite number of generators

$$
H^{*}(X ; k) \cong \Lambda_{k}\left(x_{i_{1}}, x_{i_{2}}, \ldots x_{i_{n}}\right),
$$

where the $x_{i_{j}}$ are generators in odd degrees, $\left|x_{i_{j}}\right|=i_{j}$, and $i_{j+1} \geq i_{j}$. Then when $\frac{i_{n}+\sum_{j=1}^{n} i_{j}}{i_{1}-1} \leq p$, the homology of the free loop space on $X$ is given as a graded $k$-module by

$$
H_{*}(\mathcal{L} X ; k) \cong \Lambda_{k}\left(y_{i_{1}}, y_{i_{2}}, \ldots y_{i_{n}}\right) \otimes k\left[w_{i_{1}}, w_{i_{2}}, \ldots w_{i_{n}}\right]
$$

where $\left|y_{i_{j}}\right|=i_{j}$, and $\left|w_{i_{j}}\right|=i_{j}-1$.

The case where $H^{*}(X ; k)$ is exterior on two generators had been previously considered in work of Kuribayashi and Yamaguchi [25]. Theorem 1.3 extends their result in the two generator case to a broader range of degrees, while also treating the case of more than two generators.

1.1. Organization. This paper is organized as follows. In Section 2 we recall some classical foundations for coalgebras and introduce the new notion of a $\square_{D}$-Hopf algebra over a coalgebra $D$. The definition of coHochschild homology for coalgebras is recalled in Section 3, and we prove in that section that the coHochschild homology of a coalgebra $D$ has the structure of a $\square_{D}$-bialgebra. In Section 4 we move to the topological setting, establishing the infinity categorical framework in which to discuss topological coHochschild homology, and defining coTHH. In Section 5 we use the relationship between coTHH and free loop spaces to show that under coflatness conditions the homology of a free loop space has the structure of a $\square$-Hopf algebra. In Section 6 we begin our analysis of the coBökstedt spectral sequence. We prove that under coflatness conditions the coBökstedt spectral sequence for computing coTHH $(C)$ is a spectral sequence of $\square$-Hopf algebras over the homology of $C$. Finally, in Section 7 we use this new algebraic structure on the coBökstedt spectral sequence to make explicit computations of the homology of free loop spaces.

1.2. Acknowledgments. The authors express their gratitude to the organizers of the Women in Topology II Workshop and the Banff International Research Station, where this collaboration began. We thank Vigleik Angeltveit, Ben Antieau, David Chan, Paul Goerss, Kathryn Hess, Sarah Klanderman, Maximilien Péroux, Emily Riehl, and Stephanie Ziegenhagen for helpful conversations related to this 
work. The authors also thank an anonymous referee for helpful comments. This research was supported by the National Science Foundation [DMS-1710534 and DMS2104300 to Bohmann, DMS-1810575 to Gerhardt, and DMS-1811278 to Shipley]. Some of this work was done while the second author was in residence at the Mathematical Sciences Research Institute in Berkeley, CA (supported by the National Science Foundation under grant DMS-1440140) during the Spring 2020 semester. The first and third authors would like to thank the Isaac Newton Institute for Mathematical Sciences, Cambridge, for support and hospitality during the program 'Homotopy harnessing higher structures' during which some of the work on this paper was carried out. Work during this program was supported by EPSRC grant no EP/K032208/1.

\section{2. (Co)ALGEBRAiC STRUCTURES}

Hopf algebra structures arise naturally in the study of (topological) Hochschild homology. For a commutative ring spectrum $R, \operatorname{THH}(R)$ is a Hopf algebra in the homotopy category. Further, Angeltveit and Rognes prove that under appropriate flatness conditions, the Bökstedt spectral sequence computing $H_{*}(\mathrm{THH}(R) ; k)$ is a spectral sequence of $H_{*}(R ; k)$-Hopf algebras [2]. It is then natural to ask whether the coBökstedt spectral sequence computing $H_{*}(\operatorname{coTHH}(C) ; k)$ for a coalgebra spectrum $C$ is similarly a spectral sequence of Hopf algebras. However, the usual definition of a Hopf algebra over a ring is not the correct framework for this question. The coBökstedt spectral sequence should have a multiplication and comultiplication structure over $H_{*}(C ; k)$, but $H_{*}(C ; k)$ is a coalgebra, not a ring. To study the algebraic structure in the coBökstedt spectral sequence, one needs a notion of a Hopf algebra-like structure over a coalgebra $D$. In this section we define such a structure, which we call a $\square_{D}$-Hopf algebra.

We begin by reviewing some standard definitions for coalgebras before arriving at our new constructions of $\square_{D}$-algebras, coalgebras, bialgebras, and Hopf algebras.

Definition 2.1. A coalgebra $D$ over a field $k$ is a $k$-vector space along with $k$-linear maps

$$
\triangle: D \rightarrow D \otimes D \quad \text { and } \quad \epsilon: D \rightarrow k,
$$

called the comultiplication and counit, such that the following coassociativity and counitality diagrams commute:
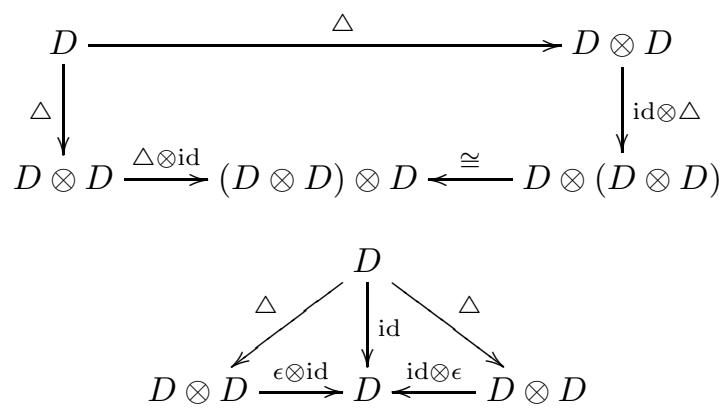

The coalgebra $D$ is called coaugmented if there is furthermore a coaugmentation $k$-linear map $\eta: k \rightarrow D$, satisfying the identities

$$
\triangle \eta=\eta \otimes \eta \quad \text { and } \quad \epsilon \eta=\mathrm{id} .
$$


We recall some classical examples of coalgebras over a field which will play an important role in this work.

Example 2.2. Let $D=k\left[w_{1}, w_{2}, \ldots\right]$, where each $w_{i}$ is in even degree, denote the $k$ coalgebra with vector space basis $\left\{w_{1}^{j_{1}} w_{2}^{j_{2}} \cdots\right\}_{j_{i} \geq 0}$, and with comultiplication given by

$$
\triangle\left(w_{i}^{j}\right)=\sum_{k}\left(\begin{array}{l}
j \\
k
\end{array}\right) w_{i}^{k} \otimes w_{i}^{j-k}
$$

on basis elements of the form $w_{i}^{j}$. This defines the comultiplication $\left.\Delta\right|_{i}$ on the underlying vector space of $k\left[w_{i}\right]$ for each $i$. To extend to all basis elements, regard $w_{i_{1}}^{j_{1}} \cdots w_{i_{n}}^{j_{n}}$ as the simple tensor $w_{i_{1}}^{j_{1}} \otimes \cdots \otimes w_{i_{n}}^{j_{n}} \in k\left[w_{i_{1}}\right] \otimes \cdots \otimes k\left[w_{i_{n}}\right]$. The comultiplication is defined by the composite

$$
\begin{aligned}
k\left[w_{i_{1}}\right] \otimes \cdots \otimes k\left[w_{i_{n}}\right] \stackrel{\left.\left.\Delta\right|_{i_{1}} \otimes \cdots \otimes \Delta\right|_{i_{n}}}{\longrightarrow} k\left[w_{i_{1}}\right] \otimes k\left[w_{i_{1}}\right] \otimes \cdots \otimes k\left[w_{i_{n}}\right] \otimes k\left[w_{i_{n}}\right] \\
\stackrel{\sigma}{\longrightarrow} k\left[w_{i_{1}}\right] \otimes \cdots \otimes k\left[w_{i_{n}}\right] \otimes k\left[w_{i_{1}}\right] \otimes \cdots \otimes k\left[w_{i_{n}}\right]
\end{aligned}
$$

where $\sigma$ is the evident permutation of the tensor factors. The counit is given by:

$$
\epsilon\left(w_{1}^{j_{1}} w_{2}^{j_{2}} \cdots\right)= \begin{cases}1 & \text { if all } j_{i}=0 \\ 0 & \text { if some } j_{i}>0\end{cases}
$$

We refer to this as the polynomial coalgebra. Some readers will find this example familiar as the underlying coalgebra of the polynomial Hopf algebra; the fact that this coalgebra extends to a Hopf algebra structure determines the comultiplication on all basis elements from its definition on the elements $w_{i}$.

Example 2.3. Let $D=\Lambda_{k}\left(x_{1}, x_{2}, \ldots\right)$, where the degree of each $x_{i}$ is odd, denote the $k$-coalgebra with vector space basis $\left\{x_{i_{1}} \cdots x_{i_{n}}\right\}_{n \geq 0, i_{1}<\cdots<i_{n}}$, and with comultiplication on $x_{i}$ given by

$$
\triangle\left(x_{i}\right)=1 \otimes x_{i}+x_{i} \otimes 1
$$

The comultiplication can be extended from $\Lambda_{k}\left(x_{i}\right)$ to all basis elements as above in Example 2.2. The counit is given by:

$$
\epsilon\left(x_{i_{1}} \cdots x_{i_{n}}\right)=0, \quad \epsilon(1)=1 .
$$

This is the cofree graded cocommutative coaugmented coalgebra on the cogenerators $\left\{x_{1}, x_{2}, \ldots\right\}$ More details about the cofree coalgebra construction may be found in [34, Chapter 12]. We refer to this as the exterior coalgebra. Again, this example may be familiar as the underlying coalgebra structure of the exterior Hopf algebra.

Definition 2.4. A graded $k$-coalgebra $D_{*}$ is connected if $D_{*}=0$ when $*<0$ and the counit map $\epsilon: D_{*} \rightarrow k$ is an isomorphism in degree zero.

Definition 2.5. Let $D$ be a coalgebra over a field $k$. A right $D$-comodule is a $k$-vector space $M$ along with a linear coaction map

$$
\rho_{M}: M \rightarrow M \otimes D
$$

such that the following coassociativity and counitality diagrams commute:
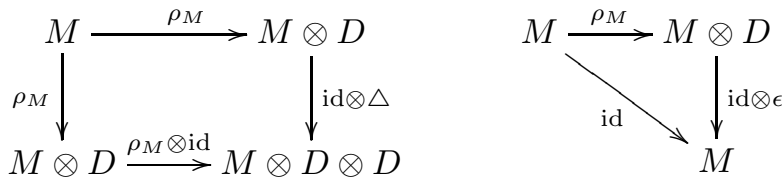
A left $D$-comodule is a $k$-vector space $N$ along with a linear coaction map

$$
\rho_{N}: N \rightarrow D \otimes N,
$$

such that coassociativity and counitality diagrams analogous to those above commute.

Definition 2.6. Let $D$ be a coalgebra over a field $k$. A $(D, D)$-bicomodule is a $k$-vector space $M$ that is both a left and right $D$-comodule, such that the following diagram commutes:

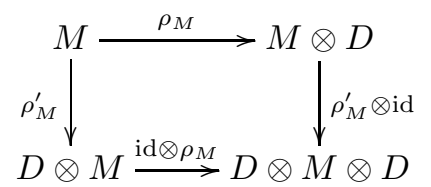

Here $\rho_{M}$ denotes the right $D$-coaction map and $\rho_{M}^{\prime}$ denotes the left $D$-coaction map.

Given a right $D$-comodule $M$ and a left $D$-comodule $N$, one can define their cotensor product $M \square_{D} N$ as follows.

Definition 2.7. Let $D$ be a coalgebra over a field $k$. Let $M$ be a right $D$-comodule and $N$ be a left $D$-comodule with coaction maps $\rho_{M}: M \rightarrow M \otimes D$ and $\rho_{N}: N \rightarrow$ $D \otimes N$. The cotensor product of $M$ and $N$ over $D, M \square_{D} N$, is defined as the equalizer in $k$-vector spaces:

$$
M \square_{D} N \longrightarrow M \otimes N \underset{\mathrm{id} \otimes \rho_{N}}{\stackrel{\rho_{M} \otimes \mathrm{id}}{\longrightarrow}} M \otimes D \otimes N .
$$

By construction, the cotensor $M \square_{D} N$ is naturally a $k$-vector space. If $M$ and $N$ are bicomodules, the resulting cotensor $M \square_{D} N$ will again be a bicomodule. Note that this relies on the fact that we are working with coalgebras over a field. For coalgebras over a general commutative ring $R$, the cotensor $M \square_{D} N$ may not be a $D$-comodule (cf. $[9,11.3])$. In fact, because we are working over a field, the category $\mathrm{BiCoMod}_{D}$ of bicomodules is an abelian category in which finite products and coproducts are given by direct sum (which agrees with Cartesian product) of $k$-vector spaces $[9,3.26]$. The cotensor $\square_{D}$ is a monoidal product on this category with unit $D$.

If $B$ and $D$ are cocommutative $k$-coalgebras, a map of cocommutative coalgebras $B \rightarrow D$ gives $B$ the structure of a $D$-comodule. There is an alternate description of $\square_{D}$ for such comodules. This is dual to observing that the pushout of commutative algebras produces the tensor product.

Observation 2.8. Given cocommutative coalgebras $B_{1}, B_{2}$, and $C$ over a field $k$, along with maps of $k$-coalgebras $f_{1}: B_{1} \rightarrow C$ and $f_{2}: B_{2} \rightarrow C$, we can view $B_{1}$ and $B_{2}$ as $C$-bicomodules. The right coaction map $\rho_{B_{1}}$, for instance, is given by

$$
\rho_{B_{1}}: B_{1} \stackrel{\triangle}{\longrightarrow} B_{1} \otimes B_{1} \stackrel{\operatorname{id} \otimes f_{1}}{\longrightarrow} B_{1} \otimes C .
$$

In this case, the pullback in the category of cocommutative $k$-coalgebras of the diagram

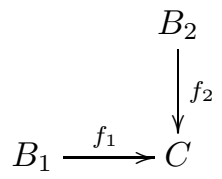


also agrees with the cotensor product $B_{1} \square_{C} B_{2}$ of $B_{1}$ and $B_{2}$ as $C$-bicomodules. One can readily verify that, since we're working over a field, the cotensor product $B_{1} \square_{C} B_{2}$ is again a cocommutative coalgebra and satisfies the universal property of the displayed pullback.

In our work it will be important to consider the exactness of the cotensor product.

Definition 2.9. Let $D$ be a coalgebra over a field $k$. A right comodule $M$ over a coalgebra $D$ is coflat if $M \square_{D}$ - is exact as a functor from left $D$-comodules to $k$-vector spaces.

For a coalgebra $D$ over a field $k$, any direct summand of a cofree $D$-comodule is coflat. In fact, in this case coflat $D$-comodules are precisely the $D$-injective comodules $[9,10.12]$, which are precisely the direct summands of cofree comodules [14].

Observe that the standard definitions of coalgebraic structures above all involve working over a base ring, which in our case we take to be the field $k$. As mentioned, the natural structures arising in coTHH also include new coalgebraic structures defined with respect to a base coalgebra. In particular, for a coalgebra $D$, we need notions of algebras, coalgebras, bialgebras, and Hopf algebras over $D$. These definitions require working with $D$-bicomodules. When $D$ is cocommutative, any $D$-comodule naturally has an induced $D$-bicomodule structure, and the examples we consider in the remainder of the paper are of this form.

Definition 2.10. Let $D$ be a cocommutative coalgebra over a field $k$. A $\square_{D}$-coalgebra $E$ is a $D$-bicomodule together with maps

$$
\triangle: E \rightarrow E \square_{D} E \quad \text { and } \quad \epsilon: E \rightarrow D
$$

of $D$-bicomodules satisfying appropriate coassociativity and counitality conditions. Explicitly, for coassociativity, we require that the following diagram commutes.

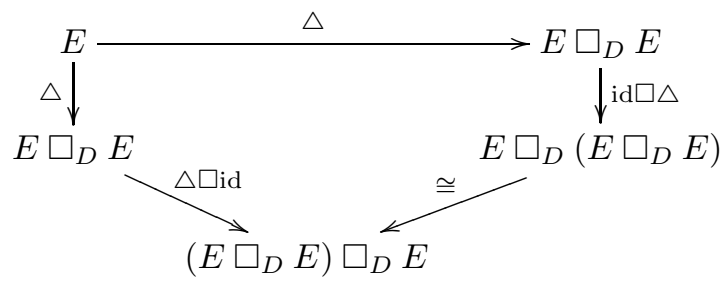

Counitality is the requirement that the following diagram commutes.

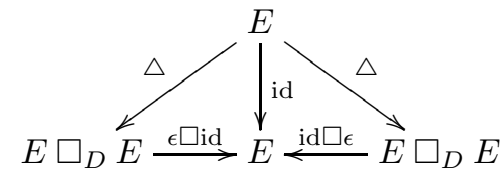

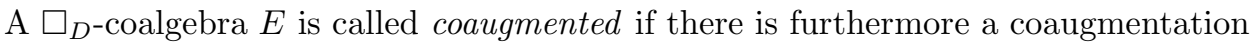
morphism $\eta: D \rightarrow E$, satisfying the identities

$$
\triangle \eta=\eta \square \eta \quad \text { and } \quad \epsilon \eta=\mathrm{id} .
$$

Definition 2.11. Let $D$ be a cocommutative coalgebra over a field $k$. A $\square_{D}$-algebra $A$ is a $D$-bicomodule together with maps of $D$-bicomodules $\mu: A \square_{D} A \rightarrow A$ and $\eta: D \rightarrow A$, satisfying the usual associativity and unitality conditions. A $\square_{D}$-algebra 
$A$ is called augmented if furthermore there is an augmentation morphism $\epsilon: A \rightarrow D$ satisfying the identities

$$
\epsilon \mu=\epsilon \square \epsilon \quad \text { and } \quad \epsilon \eta=\mathrm{id} .
$$

Definition 2.12. Let $D$ be a cocommutative coalgebra over a field $k$. A $\square_{D}$-bialgebra $H$ is a $\square_{D}$-coalgebra that is also equipped with a multiplication $\mu: H \square_{D} H \rightarrow H$ and a unit $\eta: D \rightarrow H$ that are maps of $D$-bicomodules. These must satisfy the usual associativity and unit conditions and additionally the following diagrams must commute:

(1) Compatibility of comultiplication and multiplication

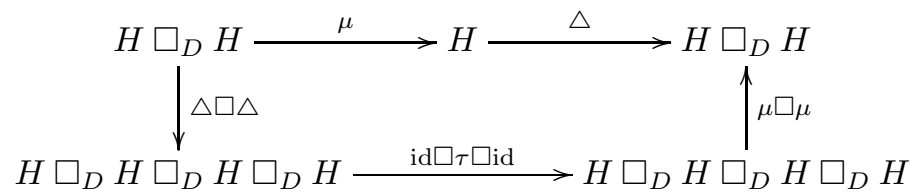

where $\tau$ is the twist.

(2) Compatibility of multiplication and counit

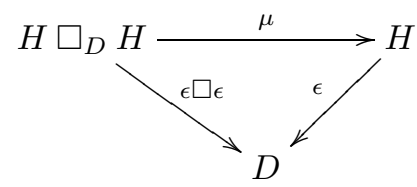

(3) Compatibility of comultiplication and unit

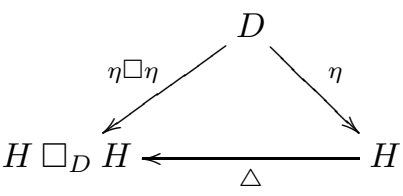

(4) Compatibility of unit and counit

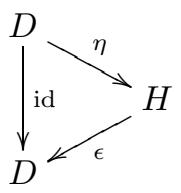

Definition 2.13. Let $D$ be a cocommutative coalgebra over a field $k$. A $\square_{D}$-Hopf algebra $H$ is a $\square_{D}$-bialgebra together with a $D$-bicomodule map $\chi: H \rightarrow H$ called the antipode making the following diagram commute:

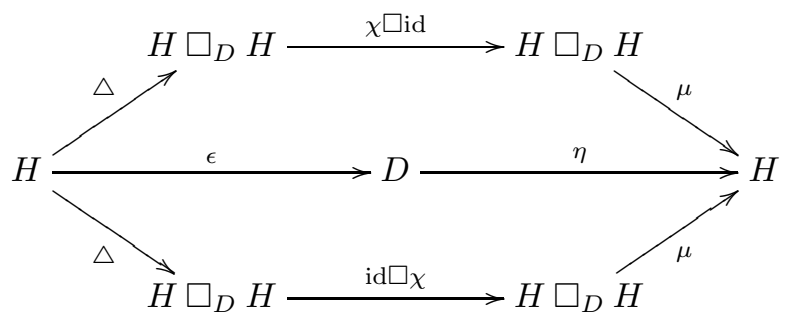


Remark 2.14. A $\square_{D}$-Hopf algebra can equivalently be defined as a Hopf monoid in the category of $D$-bicomodules. A Hopf monoid in a monoidal category is an object that has compatible monoid and comonoid structures, together with an antipode. See $[1, \S 1.2 .5]$ for more details.

We now define what it means for elements in a $\square_{D}$-Hopf algebra to be indecomposable or primitive.

Definition 2.15. Let $A$ be an augmented $\square_{D}$-algebra with augmentation $\epsilon: A \rightarrow$ $D$. Let $I A=\operatorname{ker}(\epsilon)$ denote the the augmentation ideal of $A$. We define the indecomposable elements of $A$, denoted $Q A$, by the exact sequence

$$
I A \square_{D} I A \stackrel{\mu}{\longrightarrow} I A \longrightarrow Q A \longrightarrow 0 .
$$

In other words, an element is indecomposable if it is in the kernel of the augmentation, but not in the image of the product on the augmentation ideal.

Definition 2.16. Let $E$ be a coaugmented $\square_{D}$-coalgebra, with coaugmentation $\eta: D \rightarrow E$. Let $J E$ denote the cokernel of $\eta$, and let $I E$ denote the kernel of the counit $\epsilon: E \rightarrow D$. Let $P E$ be defined by the exact sequence

$$
0 \longrightarrow P E \longrightarrow J E \stackrel{\triangle}{\longrightarrow} J E \square_{D} J E .
$$

An element $d \in I E$ is primitive if its image in $J E$ is in $P E$.

Lemma 2.17. Let $E$ be a coaugmented $\square_{D}$-coalgebra. If $e \in I E$ then

$$
\triangle(e)=e \square_{D} 1+1 \square_{D} e+\sum_{i} e_{(i)}^{\prime} \square_{D} e_{(i)}^{\prime \prime}
$$

where $\sum_{i} e_{(i)}^{\prime} \square_{D} e_{(i)}^{\prime \prime} \in I E \square_{D} I E$.

Proof. The coaugmented $\square_{D}$-coalgebra $E$ splits as $E \cong D \oplus I E$, and

$$
E \square_{D} E=\left(D \square_{D} D\right) \oplus\left(I E \square_{D} D\right) \oplus\left(D \square_{D} I E\right) \oplus\left(I E \square_{D} I E\right) .
$$

Then the statement holds because by counitality

$$
\text { id }=\left(\epsilon \square_{D} \text { id }\right) \circ \triangle=\left(\text { id } \square_{D} \epsilon\right) \circ \triangle .
$$

Note that the natural map $I E \rightarrow J E$ is an isomorphism. It follows that if $e \in I E$ is primitive, then $\triangle(e)=e \square_{D} 1+1 \square_{D} e$.

The following proposition allows us to understand $\square_{C}$-primitive elements in a $\square_{C}$-coalgebra of the form $C \otimes D$. This will be computationally useful in Section 7 .

Proposition 2.18. Let $C$ and $D$ be cocommutative coaugmented $k$-coalgebras. Then $C \otimes D$ is a $\square_{C}$-coalgebra, and an element of the form $c \otimes d \in C \otimes D$ is primitive as an element of the $\square_{C}$-coalgebra $C \otimes D$ if and only if $d$ is primitive in the $k$-coalgebra $D$.

Proof. Recall that for $C$ and $D k$-coalgebras, the tensor product $C \otimes D$ (over $k$ ) is also a $k$-coalgebra, with comultiplication given as follows.

$$
C \otimes D \stackrel{\triangle_{C} \otimes \triangle_{D}}{\longrightarrow}(C \otimes C) \otimes(D \otimes D) \stackrel{\text { id } \otimes \tau \otimes \mathrm{id}}{\longrightarrow}(C \otimes D) \otimes(C \otimes D)
$$

where $\tau$ is the twist. Note that $C \otimes D$ is a left $C$-comodule, with coaction map

$$
\psi: C \otimes D \stackrel{\triangle_{C} \otimes \mathrm{id}}{\longrightarrow} C \otimes C \otimes D .
$$

Similarly, $C \otimes D$ is a right $C$-comodule with coaction map

$$
\rho: C \otimes D \stackrel{\triangle_{C} \otimes \mathrm{id}}{\longrightarrow} C \otimes C \otimes D \stackrel{\tau^{\prime}}{\longrightarrow} C \otimes D \otimes C,
$$


where $\tau^{\prime}$ rotates the first factor to the end. The cotensor $(C \otimes D) \square_{C}(C \otimes D)$ is defined as the equalizer

$$
(C \otimes D) \square_{C}(C \otimes D) \longrightarrow(C \otimes D) \otimes(C \otimes D) \underset{\mathrm{id} \otimes \psi}{\stackrel{\rho \otimes \mathrm{id}}{\longrightarrow}}(C \otimes D) \otimes C \otimes(C \otimes D) .
$$

Observe that the comultiplication on $C \otimes D$ as a $k$-coalgebra induces a comultiplication map $C \otimes D \rightarrow(C \otimes D) \square_{C}(C \otimes D)$ by the universal property of the equalizer. In particular, the coassociativity of the comultiplication on $C$ guarantees that the diagram below commutes:

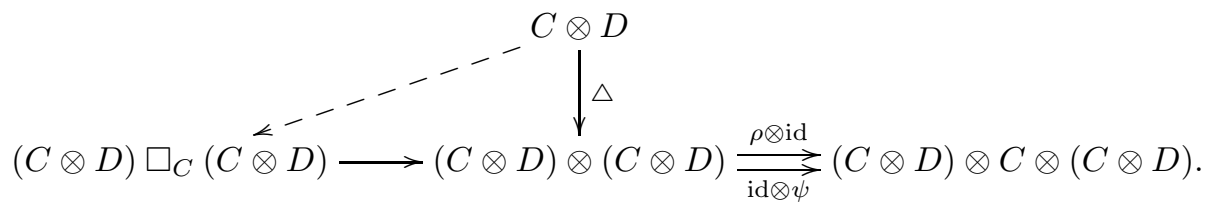

By Definition 2.16, to understand the primitive elements of $C \otimes D$ as a $\square_{C^{-}}$ coalgebra, we need to first consider the cokernel of the map $\eta: C \rightarrow C \otimes D$ defined by

$$
C \longrightarrow C \otimes k \stackrel{\operatorname{id} \otimes \eta_{D}}{\longrightarrow} C \otimes D,
$$

where $\eta_{D}$ is the coaugmentation on $D$. The cokernel of $\eta$, which we will denote $J$, is given by $J=C \otimes \operatorname{coker}\left(\eta_{D}\right)$ because we are working over the field $k$. To identify the primitive elements we must calculate the kernel of $\triangle: J \rightarrow J \square_{C} J$. This map factors as the composite

$$
J \stackrel{\triangle}{\longrightarrow}(C \otimes D) \square_{C}(C \otimes D) \rightarrow J \square_{C} J .
$$

We claim that these primitive elements are $C \otimes P D$, where $P D$ denotes the primitive elements of $D$ as a $k$-coalgebra. To see this, consider the exact sequence defining the primitive elements of $D$ as a $k$-coalgebra:

$$
0 \longrightarrow P D \longrightarrow \operatorname{coker}\left(\eta_{D}\right) \stackrel{\triangle_{D}}{\longrightarrow} \operatorname{coker}\left(\eta_{D}\right) \otimes \operatorname{coker}\left(\eta_{D}\right) .
$$

Tensoring this with $C$ over the field $k$ we have

$$
0 \longrightarrow C \otimes P D \longrightarrow C \otimes \operatorname{coker}\left(\eta_{D}\right) \stackrel{\operatorname{id} \otimes \triangle_{D}}{\longrightarrow} C \otimes\left(\operatorname{coker}\left(\eta_{D}\right) \otimes \operatorname{coker}\left(\eta_{D}\right)\right) .
$$

Noting that

$$
C \otimes\left(\operatorname{coker}\left(\eta_{D}\right) \otimes \operatorname{coker}\left(\eta_{D}\right)\right) \cong\left(C \otimes \operatorname{coker}\left(\eta_{D}\right)\right) \square_{C}\left(C \otimes \operatorname{coker}\left(\eta_{D}\right)\right),
$$

the conclusion follows.

\section{COHOCHSCHILD HOMOLOGY}

In this section, we recall the definition of coHochschild homology, and prove that under coflatness conditions, the coHochschild homology of $D$ has the structure of a $\square_{D}$-bialgebra.

Definition 3.1 (Doi [14]). Let $D$ be a cocommutative coalgebra over a field $k$. Then the coHochschild homology $\operatorname{coHH}(D)$ of $D$ is the homology of the cochain complex $C^{*}(D)$ defined by

$$
C^{n}(D)=D^{\otimes n+1}
$$


with differential $d$ given by

$$
\begin{aligned}
d\left(d_{0} \otimes \cdots \otimes d_{n}\right)=\sum_{i=0}^{n}(-1)^{i} d_{0} \otimes \cdots \otimes & \triangle\left(d_{i}\right) \otimes \cdots \otimes d_{n} \\
+ & (-1)^{n+1} \tau_{1, n+1}\left(\triangle\left(d_{0}\right) \otimes d_{1} \otimes \cdots \otimes d_{n}\right)
\end{aligned}
$$

where $\tau_{1, n+1}$ is the twist map that moves the first tensor factor in $D^{\otimes n+2}$ to the last spot.

This definition can be extended to define coHochschild homology for graded coalgebras and differential graded coalgebras, as in [19].

The cochain complex in Definition 3.1 is the (unnormalized) cochain complex of a cosimplicial $k$-module $\operatorname{coHH}^{\bullet}(D)$ defined as follows.

Definition 3.2. Let $D$ be a cocommutative coalgebra over a field $k$. The cosimplicial $k$-module $\operatorname{coHH}^{\bullet}(D)$ is given by

$$
\operatorname{coHH}^{n}(D)=D^{\otimes n+1}
$$

with cofaces

$$
\delta_{i}: D^{\otimes n+1} \rightarrow D^{\otimes n+2}, \quad \delta_{i}= \begin{cases}D^{\otimes i} \otimes \triangle \otimes D^{n-i}, & 0 \leq i \leq n, \\ \tau\left(\triangle \otimes D^{\otimes n}\right), & i=n+1,\end{cases}
$$

where $\tau$ twists the first factor to the last, and codegeneracies

$$
\sigma_{i}: D^{\otimes n+2} \rightarrow D^{\otimes n+1}, \quad \sigma_{i}=D^{\otimes i+1} \otimes \epsilon \otimes D^{\otimes n-i} \quad \text { for } 0 \leq i \leq n .
$$

Under the generalized Dold-Kan correspondence in the cosimplicial setting, the cosimplicial $k$-module $\operatorname{coHH}^{\bullet}(D)$ is sent to the normalization of the cochain complex in Definition 3.1; see [35, Corollary/Definition 8.4.3].

In order to prove that the coHochschild homology of $D$ has the structure of a $\square_{D}$-bialgebra, we first verify that some standard results from homological algebra carry over to the setting of $\square_{D}$-products. In particular, we will use versions of the Eilenberg-Zilber Theorem and the Künneth Theorem for $\square_{D}$-products.

Proposition 3.3. Let $D$ be a coalgebra over a field $k$, and let $A^{\bullet}$ and $B^{\bullet}$ be cosimplicial D-bicomodules. Then the Eilenberg-Zilber (shuffle) map induces a quasiisomorphsim of cochain complexes

$$
\text { sh: } C^{*}\left(A^{\bullet} \square_{D} B^{\bullet}\right) \rightarrow C^{*}\left(A^{\bullet}\right) \square_{D} C^{*}\left(B^{\bullet}\right) .
$$

The Alexander-Whitney map induces a quasi-inverse map

$$
A W: N^{*}\left(A^{\bullet}\right) \square_{D} N^{*}\left(B^{\bullet}\right) \rightarrow N^{*}\left(A^{\bullet} \square_{D} B^{\bullet}\right) .
$$

Here the monoidal product $\square_{D}$ of $D$-bicomodules extends to a monoidal product on cochain complexes in the usual way: for cochain complexes $A^{*}$ and $B^{*}$, the box product $A^{*} \square_{D} B^{*}$ is defined at level $n$ by

$$
\left(A^{*} \square_{D} B^{*}\right)^{n}=\bigoplus_{p+q=n} A^{p} \square_{D} B^{q} .
$$

In contrast, the monoidal product of cosimplicial $D$-bicomodules is defined levelwise. 
Proof. After applying simplicial/cosimplicial duality, this result follows from the generalized Eilenberg-Zilber theorem for an arbitrary abelian category, which is proved in [35, Section 8.5]. In more detail, the category $\operatorname{BiCoMod}_{D}^{\Delta}$ of cosimplicial $D$-bicomodules is equal to the opposite category of simplicial objects in $\operatorname{BiCoMod}_{D}^{\text {op }}$, and the category of nonnegatively graded cochain complexes in $\operatorname{BiCoMod}_{D}$ is equal to the opposite category of nonnegatively graded chain complexes in $\mathrm{BiCoMod}_{D}^{\text {op }}$. The generalized Eilenberg-Zilber theorem applies to the abelian category BiCoMod ${ }_{D}^{\text {op }}$ to produce natural shuffle and Alexander-Whitney maps in chain complexes in $\operatorname{BiCoMod}_{D}^{\text {op }}$, which then dualize to the maps above.

Proposition 3.4 (Künneth Theorem over $\square_{D}$ ). Let $D$ be a coalgebra over a field $k$ and let $A^{*}$ and $B^{*}$ be bounded below cochain complexes of D-bicomodules. Then there is a Künneth map

$$
H\left(A^{*} \square_{D} B^{*}\right) \rightarrow H\left(A^{*}\right) \square_{D} H\left(B^{*}\right) .
$$

If $A^{*}$ is a complex of coflat $D$-comodules and all $H_{i}\left(A^{*}\right)$ (or $H_{i}\left(B^{*}\right)$ ) are coflat $D$-comodules, then the Künneth map is an isomorphism.

Proof. The naturality of the Künneth theorem for cochain complexes of $k$-modules implies that we get commutative squares choosing either the left or right vertical arrows in the lower square below:

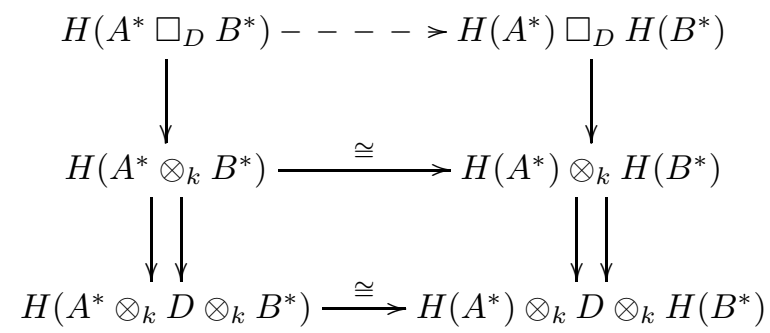

Here, to obtain the parallel maps in the left column, we view $D$ as a cochain complex concentrated in degree 0 and note that the right coaction maps $A^{n} \rightarrow A^{n} \otimes D$ assemble into a map of $k$-cochain complexes $A^{*} \rightarrow A^{*} \otimes D$; similarly for the left coaction maps on $B^{*}$. The $\square_{D}$ product $A^{*} \square_{D} B^{*}$ is the equalizer in cochain complexes of these coaction maps.

Since the the top left arrow equalizes the lower left parallel arrows in the category of cochain complexes and since homology is functorial, the top left arrow equalizes the right parallel arrows after passing through the horizontal isomorphisms. Hence there is an induced map to the equalizer of the right lower parallel arrows, which is $H\left(A^{*}\right) \square_{D} H\left(B^{*}\right)$ as shown. This is the desired map

$$
H\left(A^{*} \square_{D} B^{*}\right) \rightarrow H\left(A^{*}\right) \square_{D} H\left(B^{*}\right) .
$$

If $A^{*}$ is a complex of coflat $D$-comodules, the dual Künneth spectral sequence [15] has the form

$$
E_{2}^{p, q}=\bigoplus_{s+t=q} \operatorname{Cotor}_{D}^{p}\left(H_{s}\left(A^{*}\right), H_{t}\left(B^{*}\right)\right) \Rightarrow H_{p+q}\left(A^{*} \square_{D} B^{*}\right) .
$$

If all $H_{i}\left(A^{*}\right)$ (or all $H_{i}\left(B^{*}\right)$ ) are coflat $D$-comodules, $\operatorname{Cotor}_{D}^{p}\left(H_{s}\left(A^{*}\right), H_{t}\left(B^{*}\right)\right.$ ) is trivial for $p>0$, and this spectral sequence collapses, yielding the desired isomorphism. 
Having established these homological algebra results, we now verify that for a cocommutative coalgebra $D$ over a field, its coHochschild homology, $\operatorname{coHH}(D)$, is a $\square_{D}$-bialgebra. Note that the cosimplicial $k$-module $\operatorname{coHH}^{\bullet}(D)$ is a cosimplicial $D$-bicomodule. At level $n$, the left coaction of $D$ is given by the comultiplication on the first copy of $D$ :

$$
D \otimes\left(D^{\otimes n}\right) \stackrel{\triangle \otimes \mathrm{id}}{\longrightarrow} D \otimes D \otimes\left(D^{\otimes n}\right) .
$$

The right coaction is given by comultiplication followed by a twist:

$$
D \otimes\left(D^{\otimes n}\right) \stackrel{\triangle \otimes \mathrm{id}}{\longrightarrow} D \otimes D \otimes\left(D^{\otimes n}\right) \stackrel{\tau_{1, n+1}}{\longrightarrow} D \otimes\left(D^{\otimes n}\right) \otimes D .
$$

Proposition 3.5. Let $D$ be a cocommutative coalgebra over a field $k$. Then the coHochschild homology, $\mathrm{coHH}_{*}(D)$, is a $\square_{D}$-coalgebra.

Proof. We must define a map of $D$-comodules $\operatorname{coHH}_{*}(D) \rightarrow \operatorname{coHH}_{*}(D) \square_{D} \operatorname{coHH}_{*}(D)$. We use the generalized Eilenberg-Zilber theorem to first build this map at the cosimplicial level.

At cosimplicial level $n$, the cocommutative comultiplication $\triangle: D \rightarrow D \otimes D$ induces a map

$$
\triangle: \operatorname{coHH}^{n}(D) \rightarrow \operatorname{coHH}^{n}(D) \otimes \operatorname{coHH}^{n}(D)
$$

by applying $\triangle$ to each tensor factor and then reordering to interleave the copies of $D$. Because $D$ is coassociative and cocommutative, this map equalizes the left and right $D$-comodule action at each level and thus produces a map of $D$-comodules

$$
\operatorname{coHH}^{n}(D) \rightarrow \operatorname{coHH}^{n}(D) \square_{D} \operatorname{coHH}^{n}(D) .
$$

Hence the map of cosimplicial $k$-modules

$$
\triangle^{\bullet}: \operatorname{coHH}^{\bullet}(D) \rightarrow \operatorname{coHH}^{\bullet}(D) \otimes \operatorname{coHH}^{\bullet}(D)
$$

in fact induces a map to the equalizer

$$
\triangle^{\bullet}: \operatorname{coHH}^{\bullet}(D) \rightarrow \operatorname{coHH}^{\bullet}(D) \square_{D} \operatorname{coHH}^{\bullet}(D),
$$

recalling that limits (and colimits) in cosimplicial objects are computed levelwise.

Applying the Dold-Kan equivalence, we obtain a map of cochain complexes

$$
N^{*}\left(\operatorname{coHH}^{\bullet}(D)\right) \rightarrow N^{*}\left(\operatorname{coHH}^{\bullet}(D) \square_{D} \operatorname{coHH}^{\bullet}\right) .
$$

The generalized cosimplicial Eilenberg-Zilber theorem of Proposition 3.3 implies that the shuffle map induces a quasi-isomorphism of cochain complexes of $D$ bicomodules

$$
N^{*}\left(\operatorname{coHH}^{\bullet}(D) \square_{D} \operatorname{coHH}^{\bullet}(D)\right) \rightarrow N^{*}\left(\operatorname{coHH}^{\bullet}(D)\right) \square_{D} N^{*}\left(\operatorname{coHH}^{\bullet}(D)\right) .
$$

Composing with the map induced from $\triangle \bullet$ above induces a map on homology

$$
\operatorname{coHH}_{*}(D) \rightarrow H_{*}\left(N^{*}\left(\operatorname{coHH}^{\bullet}(D)\right) \square_{D} N^{*}\left(\operatorname{coHH}^{\bullet}(D)\right)\right) .
$$

By Proposition 3.4 above, we then have a map

$$
\operatorname{coHH}_{*}(D) \rightarrow \operatorname{coHH}_{*}(D) \square_{D} \operatorname{coHH}_{*}(D) .
$$

The counit $\epsilon: \operatorname{coHH}_{*}(D) \rightarrow D$ is the map that sends $\operatorname{coHH}_{*}(D)$ to the 0 -cochains. One can check coassociativity and counitality by hand, or we will see later in Proposition 3.8 that these conditions follow from an alternate simplicial description of this structure. 
Proposition 3.6. If $\operatorname{coHH}_{*}(D)$ is coflat over $D$, then $\operatorname{coHH}_{*}(D)$ is additionally a $\square_{D}$-algebra.

Proof. There is a cochain level product

$$
\mu: C^{*}(D) \square_{D} C^{*}(D) \rightarrow C^{*}(D),
$$

where $C^{*}(D)$ denotes the cochain complex computing $\operatorname{coHH}_{*}(D)$. Let

$$
\sum\left(d_{0} \otimes d_{1} \otimes \ldots d_{i}\right) \otimes\left(d_{0}^{\prime} \otimes d_{1}^{\prime} \otimes \ldots d_{j}^{\prime}\right) \in C^{*}(D) \square_{D} C^{*}(D)
$$

The product $\mu$ is given on each summand

$$
\left(d_{0} \otimes d_{1} \otimes \ldots d_{i}\right) \otimes\left(d_{0}^{\prime} \otimes d_{1}^{\prime} \otimes \ldots d_{j}^{\prime}\right)
$$

by the composite

$$
D^{\otimes(i+1)} \otimes D^{\otimes(j+1)} \stackrel{\mathrm{id}^{i+1} \otimes \epsilon \otimes \mathrm{id}^{j}}{\longrightarrow} D^{\otimes(i+1)} \otimes k \otimes D^{\otimes(j)} \stackrel{\cong}{\longrightarrow} D^{\otimes(i+j+1)},
$$

where $\epsilon$ is the counit in $D$. When $\operatorname{coHH}_{*}(D)$ is coflat as a $D$-comodule, by Proposition 3.4 this induces a product on coHochschild homology:

$$
\mu: \operatorname{coHH}_{*}(D) \square_{D} \operatorname{coHH}_{*}(D) \rightarrow \operatorname{coHH}_{*}(D) .
$$

There is also a unit map $\eta: D \rightarrow \operatorname{coHH}_{*}(D)$ given by the inclusion of the 0 -cochains. It is clear from the definition on cochains that the product $\mu$ is associative and unital.


algebra structure of Proposition 3.6 will make $\operatorname{coHH}_{*}(D)$ a $\square_{D}$-bialgebra when $\operatorname{coHH}_{*}(D)$ is coflat over $D$. Before proving this, we consider an alternate description of the $\square_{D}$-algebra and coalgebra structures from a cosimplicial perspective.

The identification of the coHochschild cochain complex as arising from the cosimplicial coalgebra of Definition 3.2 allows us to identify much of the structure on coHochschild homology as arising at this cosimplicial level. In particular, $\operatorname{coHH}^{\bullet}(D)$ can be viewed as a cosimplicial cotensor of $S_{\bullet}^{1}$ with the coalgebra $D$. Similar structure exists for Cartesian monoidal $\infty$-categories, as we discuss in the next section. More precisely, for a cocommutative graded $k$-coalgebra over a field $k$, the cosimplicial $k$-module of Definition 3.2 has the structure of a cosimplicial $k$-coalgebra. By inspection, this structure is in fact given by viewing coTHH. $(D)$ as the cosimplicial cotensor object $D^{S_{\bullet}^{1}}$.

With this identification, the $\square_{D}$-coalgebra structure on $\operatorname{coHH}_{*}(D)$, as defined in Proposition 3.5, is induced by the simplicial fold map $\nabla: S_{\bullet}^{1} \vee S_{\bullet}^{1} \rightarrow S_{\bullet}^{1}$. The $\square_{D}$-product structure on coHH( $\left.D\right)$, as in Proposition 3.6, is induced by a simplicial pinch map. In order to arrive at a pinch map that is indeed simplicial, however, one must use a different simplicial model of the circle. In this work we will use two different "double circle" models, denoted $d S_{\bullet}^{1}$ and $d^{\prime} S_{\bullet}^{1}$ respectively (following [2]). The double circle $d S_{\bullet}^{1}$ is given by

$$
d S_{\bullet}^{1}=\left(\Delta^{1} \amalg \Delta^{1}\right) \amalg_{\left(\partial \Delta^{1} \amalg \partial \Delta^{1}\right)} \partial \Delta^{1} .
$$

The double circle $d^{\prime} S_{\bullet}^{1}$ is given by the quotient of the double 1-simplex $d \Delta^{1}=$ $\Delta^{1} \amalg_{\Delta^{0}} \Delta^{1}$ by its two end-points, $\partial d \Delta^{1}$. It is this double circle model $d^{\prime} S_{\bullet}^{1}$ that is relevant in the current section. We revisit the model $d S_{\bullet}^{1}$ in Sections 5 and 6 .

There is a simplicial pinch map

$$
\psi: d^{\prime} S_{\bullet}^{1} \rightarrow S_{\bullet}^{1} \vee S_{\bullet}^{1}
$$





we let $d^{\prime} \mathrm{coHH}_{*}(D)$ denote the homology of the cochain complex of $k$-modules $C^{*}\left(d^{\prime} \operatorname{coHH}^{\bullet}(D)\right)$ associated to $d^{\prime} \operatorname{coHH}^{\bullet}(D)$ under the Dold-Kan correspondence. Then the simplicial pinch map $\psi$ induces a map

$$
\operatorname{coHH}_{*}(D) \square_{D} \operatorname{coHH}_{*}(D) \rightarrow d^{\prime} \operatorname{coHH}_{*}(D) .
$$

We observe that the map $\pi: d^{\prime} S_{\bullet}^{1} \rightarrow S_{\bullet}^{1}$, given by collapsing the second $\Delta^{1}$ to a point, induces an isomorphism

$$
\operatorname{coHH}_{*}(D) \rightarrow d^{\prime} \operatorname{coHH}_{*}(D) .
$$

This can be proven by dualizing the proof in [2, Lemma 2.2]. Thus, the simplicial pinch map $\psi$ induces a $\square_{D}$-product

$$
\operatorname{coHH}_{*}(D) \square_{D} \operatorname{coHH}_{*}(D) \rightarrow \operatorname{coHH}_{*}(D) .
$$

We would like to see that this product agrees with the cochain level product from Proposition 3.6.

Proposition 3.7. If $\operatorname{coHH}_{*}(D)$ is coflat over $D$, the $\square_{D}$-product

$$
\operatorname{coHH}_{*}(D) \square_{D} \operatorname{coHH}_{*}(D) \rightarrow \operatorname{coHH}_{*}(D)
$$

induced by the simplicial pinch map $\psi: d^{\prime} S_{\bullet}^{1} \rightarrow S_{\bullet}^{1} \vee S_{\bullet}^{1}$ agrees with the cochain level $\square_{D}$-product defined in Proposition 3.6.

Proof. In work of Angeltveit and Rognes [2] they prove a dual result. In the dual case, one views Hochschild homology $\mathrm{HH}_{\bullet}(A)$ as the simplicial tensor with $S_{\bullet}^{1}$, $\mathrm{HH}_{\bullet}(A)=A \otimes S_{\bullet}^{1}$. Angeltveit and Rognes then show that the simplicial pinch map induces a comultiplication on Hochschild homology

$$
\mathrm{HH}_{*}(A) \rightarrow \mathrm{HH}_{*}(A) \otimes_{A} \mathrm{HH}_{*}(A),
$$

and verify that when $\mathrm{HH}_{*}(A)$ is flat over $A$, this comultiplication agrees with the one induced by the chain level comultiplication, $\psi: C_{*}(A) \rightarrow C_{*}(A) \otimes{ }_{A} C_{*}(A)$ given by

$$
\psi\left(a_{0} \otimes a_{1} \otimes \cdots \otimes a_{q}\right)=\sum_{i=0}^{q}\left(a_{0} \otimes a_{1} \otimes \cdots \otimes a_{i}\right) \otimes_{A}\left(1 \otimes a_{i+1} \otimes \cdots \otimes a_{q}\right) .
$$

Their identification uses the shuffle equivalence

$$
\text { sh: } C_{*}(A) \otimes_{A} C_{*}(A) \rightarrow \operatorname{Ch}\left(A \otimes\left(S_{\bullet}^{1} \vee S_{\bullet}^{1}\right)\right) .
$$

The dual shuffle equivalence that we need,

$$
\operatorname{sh}: \operatorname{CoCh}\left(D^{S_{\bullet}^{1} \vee S_{\bullet}^{1}}\right) \rightarrow C^{*}(D) \square_{D} C^{*}(D)
$$

is defined in our generalized Eilenberg-Zilber theorem, Proposition 3.3. Here $C^{*}(D)$ denotes the cochain complex for $\operatorname{coHH}_{*}(D)$, as in Definition 3.1. The statement in the proposition then follows by dualizing the proof of [2, Proposition 2.3].

Proposition 3.8. If $\operatorname{coHH}_{*}(D)$ is coflat over $D$, then $\operatorname{coHH}_{*}(D)$ is a $\square_{D}$-bialgebra.

Proof. The multiplication, comultiplication, unit, and counit maps are induced by simplicial maps of the circle. In particular, there are simplicial maps:

$$
\begin{array}{ll}
\eta: S_{\bullet}^{1} \rightarrow * & \epsilon: * \rightarrow S_{\bullet}^{1} \\
\nabla: S_{\bullet}^{1} \vee S_{\bullet}^{1} \rightarrow S_{\bullet}^{1} & \psi: d^{\prime} S_{\bullet}^{1} \rightarrow S_{\bullet}^{1} \vee S_{\bullet}^{1},
\end{array}
$$


given by retraction to the basepoint, inclusion of the basepoint, fold, and pinch. Using the identification of $\operatorname{coHH}^{\bullet}(D)$ as the cosimplicial cotensor of $D$ with $S_{\bullet}^{1}$, these simplicial maps induce the following maps on $\mathrm{coHH}_{*}(D)$ :

$$
\begin{aligned}
& \eta: D \rightarrow \operatorname{coHH}_{*}(D) \\
& \epsilon: \operatorname{coHH}_{*}(D) \rightarrow D \\
& \triangle: \operatorname{coHH}_{*}(D) \rightarrow \operatorname{coHH}_{*}(D) \square_{D} \operatorname{coHH}_{*}(D) \\
& \mu: \operatorname{coHH}_{*}(D) \square_{D} \operatorname{coHH}_{*}(D) \rightarrow \operatorname{coHH}_{*}(D),
\end{aligned}
$$

By Proposition 3.7 and the preceding discussion, these maps agree with the maps described concretely in Propositions 3.5 and 3.6. It remains to check that the appropriate diagrams commute. The coassociativity and counitality of the $\square_{D^{-}}$ comultiplication $\triangle$ follow from the commutativity of the diagrams of simplicial sets below:

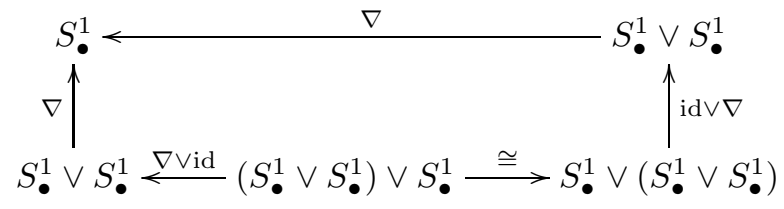

and

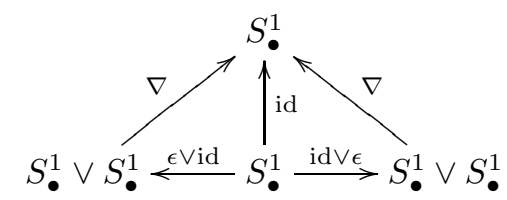

It is straightforward to verify that the product $\mu$ is associative and unital using the cochain level formulas in Proposition 3.6. One can also show this using diagrams of simplicial sets, although it requires a triple model for the circle $S^{1}$. We omit these details here.

Finally, to conclude that $\operatorname{coHH}_{*}(D)$ is a bialgebra, we need some compatibility between the $\square_{D}$-algebra structure and the $\square_{D}$-coalgebra structure, as described in Definition 2.12. The compatibility of the multiplication and comultiplication follows from the following commutative diagram of simplicial sets:

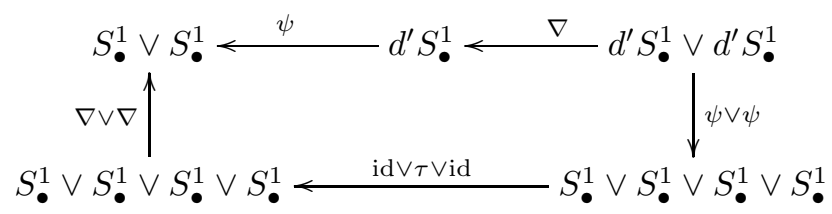

where $\tau$ is a twist map that swaps two factors. Compatibility of the multiplication and counit, compatibility of the comultiplication and unit, and compatibility of the unit and counit also follow directly from commutative diagrams of simplicial sets. Checking these compatibility conditions is left to the reader.

As an example, we compute the $\square_{\Lambda_{k}(x)}$-bialgebra structure on $\operatorname{coHH}_{*}\left(\Lambda_{k}(x)\right)$. This example will play a key role in later computations.

Proposition 3.9. Consider the exterior coalgebra $\Lambda_{k}(x)$, where $|x|=2 n+1$. There is $a \square_{\Lambda_{k}(x) \text {-coalgebra isomorphism }}$

$$
\operatorname{coHH}_{*}\left(\Lambda_{k}(x)\right) \cong \Lambda_{k}(x) \otimes k[\sigma x],
$$


where $(\sigma x)^{q}$ is the cohomology class of the cocycle $1 \otimes x \otimes x \cdots \otimes x \in C^{q}\left(\Lambda_{k}(x)\right)$. The $\square_{\Lambda_{k}(x)}$-multiplication on classes $(\sigma x)^{q}$ is given by $\mu\left((\sigma x)^{q} \otimes(\sigma x)^{s}\right)=(\sigma x)^{q+s}$.

Proof. Let $D$ denote the exterior coalgebra $\Lambda_{k}(x)$. Let $\bar{D}$ denote the kernel of the counit $\epsilon: D \rightarrow k$. Recall that the normalized coHochschild cochain complex $\left(N C^{*}(D), d\right)$ is given in degree $q$ by:

$$
N C^{q}(D)=\bigcap_{i=0}^{q-1} \operatorname{ker}\left(\sigma_{i}\right) \cong D \otimes \bar{D}^{\otimes q} .
$$

Hence,

$$
N C^{q}(D) \cong D\left\{1 \otimes x^{\otimes q}\right\}
$$

We now compute the coboundary map $d$ on the normalized cochain complex. Note that the coface maps $\delta_{i}$ for $0<i<q+1$ are zero because

$$
\triangle(x)=1 \otimes x+x \otimes 1=0 \in \bar{D} \otimes \bar{D} .
$$

Similarly,

$$
\delta_{0}\left(1 \otimes x^{\otimes q}\right)=\delta_{q+1}\left(1 \otimes x^{\otimes q}\right)=0 \in D \otimes \bar{D}^{\otimes q} .
$$

We now consider $\delta_{q+1}\left(x \otimes x^{\otimes q}\right)$. By definition,

$$
\delta_{q+1}=\tau \circ\left(\triangle \otimes \mathrm{id}^{\otimes q}\right),
$$

where $\tau$ is the rotating isomorphism that brings the front factor to the end. Note that in this graded setting, $\tau$ incorporates a sign:

$\tau\left(D_{i_{0}} \otimes D_{i_{1}} \otimes \cdots \otimes D_{i_{q+1}}\right)=(-1)^{i_{0}\left(i_{1}+i_{2}+\cdots+i_{q+1}\right)}\left(D_{i_{1}} \otimes D_{i_{2}} \otimes \cdots \otimes D_{i_{q+1}} \otimes D_{i_{0}}\right)$.

Thus, $\delta_{q+1}\left(x \otimes x^{\otimes q}\right)=(-1)^{q}\left(1 \otimes x^{q+1}\right)$ in $D \otimes \bar{D}^{\otimes(q+1)}$, and hence the sum

$$
\delta_{0}\left(x \otimes x^{\otimes q}\right)+(-1)^{q+1} \delta_{q+1}\left(x \otimes x^{\otimes q}\right)
$$

is zero. From these calculations of coface maps we conclude that $d=0$ on $N C^{q}(D)$, so

$$
\operatorname{coHH}_{q}\left(\Lambda_{k}(x)\right) \cong \Lambda_{k}(x)\left\{1 \otimes x^{\otimes q}\right\} .
$$

We write $\sigma x$ for the class $1 \otimes x$ and $(\sigma x)^{q}$ for the class $1 \otimes x^{\otimes q}$. From the description of the comultiplication in Proposition 3.5 it then follows that there is an isomorphism of $\square_{\Lambda_{k}(x) \text {-coalgebras }}$

$$
\operatorname{coHH}_{*}\left(\Lambda_{k}(x)\right) \cong \Lambda_{k}(x) \otimes k[\sigma x] .
$$

The formula for multiplication on the classes $(\sigma x)^{q}$ follows directly from the formulas for $\square$-multiplication in Proposition 3.6.

\section{Coalgebra spectra And topological COHochschild homology}

In this section we establish the infinity categorical framework in which to discuss cocommutative coalgebra spectra and cocommutative $H k$-coalgebras. Recall that there is a symmetrical monoidal $\infty$-category of spectra, which we denote Spec. We denote the symmetric monoidal $\infty$-category of $H k$-modules by $\operatorname{Mod}_{H k}$. As dual objects to algebras, coalgebras are then defined to be algebras in the opposite category. 
Definition 4.1 (An instance of [26, Definition 3.1.1]). A cocommutative coalgebra spectrum $C$ is a commutative algebra in the opposite $\infty$-category $\mathrm{Spec}^{\mathrm{op}}$. The $\infty$-category of cocommutative coalgebra spectra is defined to be $\left(\mathrm{CAlg}\left(\mathrm{Spec}^{\mathrm{op}}\right)\right)^{\mathrm{op}}$, that is, the opposite category of the category of commutative algebras in $\mathrm{Spec}^{\mathrm{op}}$. We denote this $\infty$-category by $\mathrm{CoCAlg}(\mathrm{Spec})$.

Similarly, there is an $\infty$-category of cocommutative $H k$-coalgebras.

Definition 4.2 ([26, Definition 3.1.1]). A cocommutative Hk-coalgebra is a commutative algebra in the opposite $\infty$-category $\operatorname{Mod}_{H k}^{\mathrm{op}}$. The $\infty$-category of cocommutative $H k$-coalgebras is defined to be $\left(\mathrm{CAlg}\left(\operatorname{Mod}_{H k}^{\mathrm{op}}\right)\right)^{\mathrm{op}}$. We denote this $\infty$-category by $\operatorname{CoCAlg}\left(\operatorname{Mod}_{H k}\right)$.

Remark 4.3. Note that in a classical category $\mathcal{C}$, the opposite category of commutative algebras in $\mathcal{C}^{\mathrm{op}}$ is the category of cocommutative coalgebras in $\mathcal{C}$.

In [26, Proposition 3.1.4], Lurie shows that under presentability conditions on $\mathcal{C}$ and accessibility conditions on the monoidal product, the $\infty$-category of cocommutative coalgebra objects in $\mathcal{C}$ is presentable. This applies to the $\infty$-categories of cocommutative $H k$-coalgebra spectra and of cocommutative coalgebra spectra. In particular, these categories have all small limits and colimits, and colimits of cocommutative $(H k)$-coalgebras are preserved by the forgetful functor from cocommutative $(H k)$-coalgebra spectra to spectra.

However, limits of cocommutative coalgebra spectra in general do not agree with limits in underlying spectra. This is essentially because the smash product of spectra doesn't play nice with limits, in contrast to colimits.

Further results of Lurie's Higher Algebra show that the $\infty$-categories of coalgebra spectra of Definitions 4.1 and 4.2 are symmetric monoidal.

Proposition 4.4. The $\infty$-categories $\mathrm{CoCAlg}(\mathrm{Spec})$ and $\mathrm{CoCAlg}\left(\mathrm{Mod}_{H k}\right)$ have Cartesian symmetric monoidal structures under which the (finite) Cartesian product is given on objects by the smash product of spectra.

Proof. As observed in [27, Remark 2.4.2.7], if $\mathcal{C}$ is a symmetric monoidal $\infty$ category, there is an induced symmetric monoidal structure on $\mathcal{C}^{\text {op }}$. Just as in the classical case, as discussed in $[27, \S 2.4 .3]$, this duality takes Cartesian symmetric monoidal structures to coCartesian symmetric monoidal structures. Hence, it suffices to prove that $\mathrm{CAlg}\left(\mathrm{Spec}^{\mathrm{op}}\right)$ and $\mathrm{CAlg}\left(\mathrm{Mod}_{H k}^{\mathrm{op}}\right)$ have the structure of coCartesian symmetric monoidal $\infty$-categories. This result, in turn, is [27, Proposition 3.2.4.7], which shows that for commutative algebras, the underlying symmetric monoidal product is the coproduct.

Given a cocommutative coalgebra $C \in \mathrm{CoCAlg}$ (Spec), one might ask for a nice $\infty$-category of comodules over $C$, in which to define the analogs of $\square_{C}$-coalgebras and the related algebraic structures of Section 2. While the dual construction of $\infty$ categories of modules over algebras has been extensively developed by Lurie [27], these constructions do not readily dualize because the smash product in spectra does not commute with totalization. Hence we do not generally have a suitable symmetric monoidal $\infty$-category of $C$-comodules. In later sections, we largely avoid this point by working in the special case of $H k$-coalgebras, where the Dold-Kan correspondence allows us to transfer questions to the realm of (co)chain complexes. This setting is also the subject of Péroux's rigidification results [31]. 
For a general cocommutative coalgebra $C$, we can also recover an analog of the structure of a $\square_{C}$-coalgebra via the viewpoint of Observation 2.8, which remarks that the box-product $B_{1} \square_{C} B_{2}$ of coalgebras over $C$ can be defined via pullback over $C$.

Proposition 4.5. Let $C \in \mathrm{CoCAlg}(\mathrm{Spec})$. Then the over category $\mathrm{CoCAlg}(\mathrm{Spec}) / C$ is a Cartesian monoidal $\infty$-category where the monoidal product $B_{1} \square_{C} B_{2}$ of objects $B_{1} \rightarrow C$ and $B_{2} \rightarrow C$ is the pullback in $\mathrm{CoCAlg}(\mathrm{Spec}):$

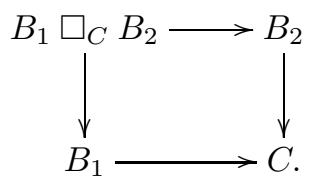

Hence every object of $\mathrm{CoCAlg}(\mathrm{Spec})_{/ C}$ is a cocommutative $\square_{C}$-coalgebra spectrum.

Proof. For concreteness, we use Joyal's definition of the $\infty$-category $\mathrm{CoCAlg}(\mathrm{Spec}) / C$ of cocommutative coalgebra spectra over $C$ [21]. That is, we view $C$ as a diagram $C: \mathbb{1} \rightarrow \mathrm{CoCAlg}(\mathrm{Spec})$, where $\mathbb{1}$ is the category with one object and only the identity morphism. Then $\mathrm{CoCAlg}(\mathrm{Spec}) / C$ is the $\infty$-category defined by the property that for a simplicial set $Y$,

$$
\operatorname{Map}\left(Y, \operatorname{CoCAlg}(\operatorname{Spec})_{/ C}\right) \simeq \operatorname{Map}_{\mathbb{1}}(Y \star \mathbb{1}, \operatorname{CoCAlg}(\operatorname{Spec})) .
$$

The subscript on the right-hand side indicates that we consider only those maps $f: Y \star \mathbb{1} \rightarrow \operatorname{CoCAlg}(\mathrm{Spec})$ such that $\left.f\right|_{\mathbb{1}}$ is $C$. The $\infty$-category $Y \star \mathbb{1}$ is the join of the simplicial sets $Y$ and $\mathbb{1}$, as defined in [21]. Since $\mathbb{1}$ is a single point, this operation produces the "cocone" on an $\infty$-category $Y$.

Since the category $\mathrm{CoCAlg}(\mathrm{Spec})$ is defined as the opposite of $\mathrm{CAlg}\left(\mathrm{Spec}^{\mathrm{op}}\right)$, by passing to opposite categories, it suffices to show that the under category

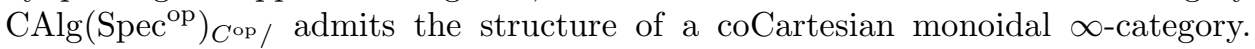
More precisely, for an arbitrary simplicial set $Y$, the join/slice adjunction [32, Proprosition 4.2.5] and op-duality provide a string of equivalences

$$
\begin{aligned}
\operatorname{Map}\left(Y, \operatorname{CoCAlg}(\operatorname{Spec})_{/ C}\right) & \simeq \operatorname{Map}_{\mathbb{1}}(Y \star \mathbb{1}, \operatorname{CoCAlg}(\operatorname{Spec})) \\
& \simeq \operatorname{Map}_{\mathbb{1}}\left(\mathbb{1} \star\left(Y^{\mathrm{op}}\right), \operatorname{CAlg}\left(\operatorname{Spec}^{\mathrm{op}}\right)\right) \\
& \simeq \operatorname{Map}\left(Y^{\mathrm{op}}, \operatorname{CAlg}\left(\operatorname{Spec}^{\mathrm{op}}\right)_{C^{\mathrm{op}} /}\right) \\
& \simeq \operatorname{Map}\left(Y,\left(\operatorname{CAlg}\left(\operatorname{Spec}^{\mathrm{op}}\right)_{\left.C^{\mathrm{op}} /\right)^{\mathrm{op}}}\right)\right.
\end{aligned}
$$

which shows that $\mathrm{CAlg}\left(\mathrm{Spec}^{\mathrm{op}}\right)_{C^{\mathrm{op}} /}$ is the opposite of $\mathrm{CoCAlg}(\mathrm{Spec}) / C$.

In [27, Section 2.4.3], Lurie defines an $\infty$-operad $\mathcal{C}^{\amalg}$ on an arbitrary $\infty$-category $\mathcal{C}$ and shows this is a symmetric monoidal $\infty$-category structure on $\mathcal{C}$ if and only if $\mathcal{C}$ admits finite coproducts. In the case of the under category $\mathrm{CAlg}\left(\mathrm{Spec}^{\mathrm{op}}\right)_{C^{\mathrm{op}} / \text {, }}$ coproducts are given by pushouts in $\mathrm{CAlg}\left(\mathrm{Spec}^{\mathrm{op}}\right)$, which in turn are pullbacks in $\mathrm{CoCAlg}(\mathrm{Spec})$ by [32, Proposition 12.1.7]. Since $\mathrm{CoCAlg}(\mathrm{Spec})$ is complete, the necessary pullbacks exist. Thus $\mathrm{CoCAlg}(\mathrm{Spec})_{/ C}$ is a Cartesian monoidal $\infty$ category.

Finally, to justify the statement that every object in $\mathrm{CoCAlg}(\mathrm{Spec})_{/ C}$ is a cocommutative coalgebra over $C$, observe that the $\infty$-category

$$
\mathrm{CoCAlg}\left(\mathrm{CoCAlg}(\mathrm{Spec})_{C}\right)
$$


of cocommutative coalgebras in $\mathrm{CoCAlg}(\mathrm{Spec})_{/ C}$ is the opposite category of

$$
\mathrm{CAlg}\left(\left(\mathrm{CoCAlg}(\mathrm{Spec})_{C}\right)^{\text {op }}\right)
$$

of commutative algebras in the opposite category, as in [26, Definition 3.1.1] and Definitions 4.1 and 4.2. Since $\left(\mathrm{CoCAlg}(\mathrm{Spec})_{/ C}\right)^{\text {op }}$ is coCartesian monoidal, by [27, Corollary 2.4.3.10], every object is a commutative algebra object, and hence every object of $\mathrm{CoCAlg}(\mathrm{Spec})_{/ C}$ is a cocommutative coalgebra object.

We next describe the topological coHochschild homology of a coalgebra spectrum in this framework. We begin with some basic recollections about Cartesian monoidal $\infty$-categories.

Let $\mathcal{C}$ be a Cartesian monoidal $\infty$-category; in particular this implies $\mathcal{C}$ admits finite products. For a finite set $X$ and an object $C \in \mathcal{C}$, the $X$-fold product $\prod_{X} C$ provides a model for the categorical cotensor $C^{X}$ : we have an equivalence of mapping spaces

$$
\operatorname{Map}_{\mathcal{C}}\left(D, \prod_{X} C\right) \simeq\left(\operatorname{Map}_{\mathcal{C}}(D, C)\right)^{X}
$$

Indeed, if $\mathcal{C}$ admits $X$-fold products for an arbitrary set $X$, we may similarly model a cotensor $C^{X}$ as a product.

This construction is contravariantly functorial in $X$, in the following sense. Suppose $\mathcal{C}$ admits products indexed by any set $X \in$ Set. We may then obtain a map of $\infty$-categories Set ${ }^{\text {op }} \stackrel{C^{-}}{\longrightarrow} \mathcal{C}$. As shown in [32, Proposition 1.1.11], there is an adjunction

$$
\operatorname{sSet}\left(\operatorname{Set}^{\text {op }}, \mathcal{C}\right) \cong \operatorname{Cat}\left(\mathrm{Set}^{\mathrm{op}}, h \mathcal{C}\right)
$$

where $h \mathcal{C}$ is the homotopy category of $\mathcal{C}$ and we are, as usual, omitting notation for the nerve of the category Set $^{\text {op }}$ on the left. By [32, Lemma 2.3.3] for a set $X$, $X$-fold products in $\mathcal{C}$ are also products in $h \mathcal{C}$, and so $h \mathcal{C}$ admits $X$-fold products. The usual argument from the universal properties of products then shows that the assignment $X \mapsto C^{X}$ is a contravariant functor $\mathrm{Set}^{\mathrm{op}} \rightarrow h \mathcal{C}$; by adjunction we thus have the desired functor of $\infty$-categories.

Given a simplicial set $X_{\bullet}$ and an object $C$ in an $\infty$-category admitting products, we obtain a $\Delta$-shaped diagram in $C$ via the composite

$$
\Delta \stackrel{X_{\bullet}^{\text {op }}}{\longrightarrow} \text { Set }^{\text {op }} \stackrel{C^{-}}{\longrightarrow} \mathcal{C} .
$$

This is a cosimplicial object in $\mathcal{C}$ which we denote by $C^{X}$ and which we view as the "cosimplicial cotensor" of $X$ • and $C$. When $\mathcal{C}$ is complete, we may take the limit $\lim _{\Delta} C^{X}$ to obtain an object of $\mathcal{C}$. We sometimes use the notation $C^{X}=\lim _{\Delta} C^{X} \cdot$ where we remove the $\bullet$ to emphasize that it is a single object of $\mathcal{C}$, rather than a cosimplicial object. This limit can alternately be described as the totalization of the cosimplicial object $C^{X}$.

In the case where $X_{\bullet}$ is the simplicial set $\Delta_{\bullet}^{1}$, this process gives a "resolution" of the object $C$.

Lemma 4.6. Let $\mathcal{C}$ be a Cartesian monoidal $\infty$-category. For an object $C \in \mathcal{C}$, let $\Delta \rightarrow \mathcal{C}$ be the cosimplicial object of $\mathcal{C}$ given by

$$
\Delta \stackrel{\left(\Delta_{\bullet}^{1}\right)^{\mathrm{op}}}{\longrightarrow} \operatorname{Set}^{\mathrm{op}} \stackrel{C^{-}}{\longrightarrow} \mathcal{C} .
$$

Then $C \simeq \lim _{\Delta} C^{\Delta_{\bullet}^{1}}$. 
Proof. The simplicial set $\Delta_{\bullet}^{1}$ is the representable functor $\Delta(-,[1])$. This extends to a split augmented simplicial object $\Delta_{\perp}^{\mathrm{op}} \rightarrow$ Set with "extra degeneracies." Here $\Delta_{\perp}$ is the category obtained from $\Delta$ by adding an "extra degeneracy" $\sigma^{-1}:[n+1] \rightarrow[n]$ for each $n$, including a coaugmentation [0] $\rightarrow[-1]$. The inclusion functor $\Delta \rightarrow \Delta_{\perp}$ has a right adjoint [32, Example B.5.2] and the fact that $\Delta_{\text {. }}^{1}$ extends to a presheaf on $\Delta_{\perp}$ follows from the fact that the representing object [1] is in the image of the right adjoint.

We thus obtain a split coaugmented cosimplicial object

$$
\Delta_{\perp} \rightarrow \operatorname{Set}^{\text {op }} \stackrel{C^{-}}{\longrightarrow} \mathcal{C}
$$

that restricts to $C^{\Delta_{\bullet}^{1}}$. By [32, Proposition 2.3.15], any cosimplicial object that admits a splitting and coaugmentation has a limit given by the coaugmentation - that is, by evaluation at $[-1] \in \Delta_{\perp}$. In this case, by construction the coaugmentation is simply the 1-fold product of $C$, meaning the limit is $C$ itself.

Since the $\infty$-category $\mathrm{CoCAlg}(\mathrm{Spec})$ is complete and cocomplete, the totalization of any of the cosimplicial objects $C^{X}$ exists. In discussing coalgebras, however, we frequently work at the cosimplicial level rather than passing all the way to the totalization.

Definition 4.7. Let $S_{\bullet}^{1}$ be the standard model of the simplicial circle as $\Delta_{\bullet}^{1} / \partial \Delta_{\bullet}^{1}$. Let $C$ be in $\mathrm{CoCAlg}(\mathrm{Spec})$. Then there is a cosimplicial cocommutative coalgebra spectrum $\operatorname{coTHH}^{\bullet}(C)$ defined by

$$
\Delta \stackrel{\left(S_{\bullet}^{1}\right)^{\text {op }}}{\longrightarrow} \operatorname{Set}^{\text {op }} \stackrel{C^{-}}{\longrightarrow} \text { CoCAlg}(\mathrm{Spec}) .
$$

The forgetful functor $\mathrm{CoCAlg}(\mathrm{Spec}) \rightarrow \mathrm{Spec}$ allows us to view $\operatorname{coTHH}^{\bullet}(C)$ simply as a cosimplicial spectrum as well. Viewing $\operatorname{coTHH}^{\bullet}(C)$ as a cosimplicial cotensor will allow us to induce structure on $\operatorname{coTHH}^{\bullet}(C)$ via maps of the simplicial circle $S_{\bullet}^{1}$, as we did for $\operatorname{coHH}^{\bullet}(D)$ in Section 3 .

Definition 4.8. Given a cocommutative coalgebra spectrum $C$, define the spectrum $\operatorname{coTHH}(C)$ to be the (homotopy) limit in spectra of the cosimplicial spectrum constructed in Definition 4.7:

$$
\operatorname{coTHH}(C)=\lim _{\Delta} \operatorname{coTHH}^{\bullet}(C)
$$

That is, $\operatorname{coTHH}(C)$ is the totalization in spectra of $\operatorname{coTHH}^{\bullet}(C)$.

Remark 4.9. In the case where $C$ is a coalgebra spectrum in one of the standard model categories of spectra, Definition 4.7 reproduces the cosimplicial spectrum $\operatorname{coTHH}^{\bullet}(C)$ whose totalization defined $\operatorname{coTHH}(C)$ in [4, Definition 2.2] and in [20]. Hence Definition 4.8 agrees with the construction of $\operatorname{coTHH}(C)$ in [4] and [20].

The essential point is that in coalgebra spectra, the comultiplication $C \rightarrow C \wedge C$ is the universal diagonal map from an object to its two-fold Cartesian productthis is why the symmetric monoidal smash product on coalgebras is the Cartesian product. Similarly, the empty product is the unit object $S$ for $\wedge$ and the map to this terminal object is the counit on $C$. One then checks that the face and degeneracy maps of $S_{\bullet}^{1}$ induce the coface and codegeneracy maps of [4, Definition 2.2]. See the discussion in $[4, \S 4]$ for more details on this identification. 
Remark 4.10. An alternate way of viewing $\operatorname{coTHH}(C)$ and the cyclic cobar complex coTHH $^{\bullet}$ in an $\infty$-categorical framework is supplied by Bayındır-Péroux [3] who use it to analyze duality relating $\mathrm{THH}$ and coTHH.

Remark 4.11. In Definition 4.8, taking the limit in spectra - as opposed to in cocommutative coalgebra spectra-may seem unnatural, especially to those familiar with the dual case, where McClure, Schwänzl, and Vogt [29] prove that for a commutative ring spectrum $A$ there is an equivalence of commutative ring spectra $\operatorname{THH}(A) \simeq A \otimes S^{1}$. In a simplicial model category of coalgebra spectra, the totalization of $\operatorname{coTHH}^{\bullet}(C)$ would produce the cotensor of $C$ with $S_{\bullet}^{1}$. However, in contrast to the situation for geometric realization of (commutative) algebra spectra, totalization in cocommutative coalgebra spectra does not generally agree with totalization in spectra. The key difference, of course, is that smash product of spectra commutes with geometric realization but not with totalization. Hence $\operatorname{Tot}_{\mathrm{CoCAlg}(\mathrm{Spec})}\left(\operatorname{coTHH}^{\bullet}(C)\right) \not \operatorname{coTHH}(C)$.

Totalizing in spectra in Definition 4.8 means that our discussion of coTHH in this paper agrees with that of [4] and extends the work of $[19,20]$. In particular, the coBökstedt spectral sequence defined in [4], and further discussed in Sections 6 and 7 of this paper, arises from the Bousfield-Kan spectral sequence for totalization in spectra; the results of [4] would not apply to a totalization in cocommutative coalgebra spectra.

Furthermore, while the present work focuses on the case of cocommutative coalgebra spectra, the construction of coTHH generalizes that of coHH, which can be applied to not-necessarily-cocommutative coalgebras, as is laid out in [4]. Given that totalization in cocommutative coalgebras, coassociative coalgebras, and underlying spectra need not agree, the uniform way to produce coTHH for all flavors of coalgebra is by totalizing in underlying spectra.

\section{Coalgebra structure And free loop spaces}

The definition of coTHH as a totalization in spectra, rather than in coalgebra spectra, means that many of the good formal properties enjoyed by THH do not necessarily carry over to this dual setting. At heart, this issue arises from the inevitable failure of totalization to commute with smash product. This means that even for a cocommutative coalgebra spectrum $C$, we cannot easily show that $\operatorname{coTHH}(C)$ is itself a coalgebra spectrum, for example. However, for the important example of suspension spectra, coTHH is much better behaved. In the case of a simply connected space $X, \operatorname{coTHH}\left(\Sigma_{+}^{\infty} X\right)$ is coalgebra spectrum, namely, as shown by Malkiewich [28] and Hess-Shipley [20], $\operatorname{coTHH}\left(\Sigma_{+}^{\infty} X\right) \simeq \Sigma_{+}^{\infty} \mathcal{L} X$. This identification, on which we elaborate in the following lemma, means that we can obtain significantly more algebraic structure on coTHH in the suspension spectrum case than in general.

Lemma 5.1. Let $X$ be a simply connected space. Then $\operatorname{coTHH}\left(\Sigma_{+}^{\infty} X\right)$ has the structure of a coalgebra spectrum with comultiplication induced by the fold map on $S_{\bullet}^{1} \amalg S_{\bullet}^{1}$.

Proof. Let $X$ be a simply connected space. Consider the cosimplicial space $X^{S_{\bullet}^{1} \amalg S_{\bullet}^{1}}$. By inspection, we have an isomorphism of cosimplicial spaces

$$
X^{S_{\bullet}^{1} \amalg S_{\bullet}^{1}} \cong X^{S_{\bullet}^{1}} \times X^{S_{\bullet}^{1}} .
$$


Since totalization commutes with products, we find

$$
\operatorname{Tot}_{\mathrm{Top}}\left(X^{S_{\bullet}^{1} \amalg S_{\bullet}^{1}}\right) \cong \operatorname{Tot}_{\mathrm{Top}}\left(X^{S_{\bullet}^{1}}\right) \times \operatorname{Tot}_{\mathrm{Top}}\left(X_{\bullet}^{S_{\bullet}^{1}}\right) .
$$

The fold map $\nabla: S_{\bullet}^{1} \amalg S_{\bullet}^{1} \rightarrow S_{\bullet}^{1}$ induces a morphism of simplicial spaces $X^{S_{\bullet}^{1}} \rightarrow$ $X^{S_{\bullet}^{1} \amalg S_{\bullet}^{1}}$, which, after totalization, induces the diagonal map

$$
\operatorname{Tot}_{\mathrm{Top}}\left(X^{S_{\bullet}^{1}}\right) \rightarrow \operatorname{Tot}_{\mathrm{Top}}\left(X^{S_{\bullet}^{1}}\right) \times \operatorname{Tot}_{\mathrm{Top}}\left(X^{S_{\bullet}^{1}}\right) .
$$

Since totalization $\operatorname{Tot}_{\mathrm{Top}}\left(X^{S_{\bullet}^{1}}\right)$ can be identified with the free loop space $\mathcal{L} X$ by [12], this map is the diagonal map $\mathcal{L} X \rightarrow \mathcal{L} X \times \mathcal{L} X$.

Malkiewich [28] shows that there is a natural map

$$
\Sigma_{+}^{\infty}\left(\operatorname{Tot}_{\mathrm{Top}}\left(X^{S_{\bullet}^{1}}\right)\right) \rightarrow \operatorname{Tot}_{\text {Spec }}\left(\Sigma_{+}^{\infty}\left(X^{S_{\bullet}^{1}}\right)\right)
$$

where on the right-hand side we have taken the suspension spectrum at each cosimplicial level of the cosimplicial space $X^{S_{\bullet}^{1}}$. Note that at cosimplicial level $n$, we may identify the suspension spectrum $\Sigma_{+}^{\infty}\left(X^{\times n}\right)$ with $\left(\Sigma_{+}^{\infty} X\right)^{\wedge n}$ and thus this right-hand cosimplicial spectrum is $\operatorname{coTHH}^{\bullet}\left(\Sigma_{+}^{\infty} X\right)$. Malkiewich further shows that this map is an equivalence (after Reedy-fibrantly replacing on the right). In fact, this map fits into a diagram



The lower horizontal map is also an equivalence, as we can see via the identification of $X^{S_{\bullet}^{1} \amalg S_{\bullet}^{1}}$ with $(X \times X)^{S_{\bullet}^{1}}$, to which Malkiewich's result applies. Using the above identifications, we further obtain the equivalence

$$
\begin{aligned}
\operatorname{Tot}_{\mathrm{Spec}}\left(\Sigma_{+}^{\infty}\left(X^{S_{\bullet}^{1} \amalg S_{\bullet}^{1}}\right)\right) & \simeq \Sigma_{+}^{\infty} \operatorname{Tot}_{\mathrm{Top}}\left(X^{S_{\bullet}^{1} \amalg S_{\bullet}^{1}}\right) \\
& \simeq \Sigma_{+}^{\infty}\left(\operatorname{Tot}_{\mathrm{Top}}\left(X^{S_{\bullet}^{1}}\right) \times \operatorname{Tot}_{\mathrm{Top}}\left(X^{S_{\bullet}^{1}}\right)\right) \\
& \simeq \Sigma_{+}^{\infty} \operatorname{Tot}_{\mathrm{Top}}\left(X^{S_{\bullet}^{1}}\right) \wedge \Sigma_{+}^{\infty} \operatorname{Tot}_{\mathrm{Top}}\left(X^{S_{\bullet}^{1}}\right) \\
& \simeq \operatorname{Tot}_{\mathrm{Spec}}\left(\Sigma_{+}^{\infty}\left(X^{S_{\bullet}^{1}}\right)\right) \wedge \operatorname{Tot}_{\mathrm{Spec}}\left(\Sigma_{+}^{\infty}\left(X^{S_{\bullet}^{1}}\right)\right) .
\end{aligned}
$$

Thus the right vertical map in the diagram is a comultiplication of spectra

$$
\triangle: \operatorname{coTHH}\left(\Sigma_{+}^{\infty} X\right) \rightarrow \operatorname{coTHH}\left(\Sigma_{+}^{\infty} X\right) \wedge \operatorname{coTHH}\left(\Sigma_{+}^{\infty} X\right)
$$

and in fact agrees with the canonical comultiplication we obtain on $\operatorname{coTHH}\left(\Sigma_{+}^{\infty} X\right)$ from the diagonal map after using the identification $\operatorname{coTHH}\left(\Sigma_{+}^{\infty} X\right) \simeq \Sigma_{+}^{\infty} \mathcal{L} X$.

We additionally have a counit map $\operatorname{coTHH}\left(\Sigma_{+}^{\infty} X\right) \rightarrow \operatorname{coTHH}\left(\Sigma_{+}^{\infty} *\right)$ that arises from the collapse map $\pi: X_{+} \rightarrow *_{+}=S^{0}$; note that $\operatorname{coTHH}\left(\Sigma_{+}^{\infty} *\right)=\operatorname{coTHH}(S) \simeq$ $S$ either from Malkiewich's result about free loop spaces or from the observation that $\operatorname{coTHH}^{\bullet}(S)$ is the constant cosimplicial spectrum at $S$. The axioms for a coalgebra spectrum thus can be verified at the cosimplicial level.

For example, we show counitality explicitly. At the cosimplicial level, the composite

$$
X^{S_{\bullet}^{1}} \stackrel{\nabla}{\longrightarrow} X^{S_{\bullet}^{1} \amalg S_{\bullet}^{1}} \simeq X^{S_{\bullet}^{1}} \times X^{S_{\bullet}^{1}} \stackrel{\pi \times \text { id }}{\longrightarrow} *^{S_{\bullet}^{1}} \times X^{S_{\bullet}^{1}} \simeq X^{S_{\bullet}^{1}}
$$

is the identity map. After passing to suspension spectra and totalizing, we obtain the left counitality condition. 
We now make two important observations following from the identifications above.

Remark 5.2. In the case of the cosimplicial spectrum $\left(\Sigma_{+}^{\infty} X\right)^{S_{\bullet}^{1}}$, totalization commutes with the smash product

$$
\operatorname{Tot}\left(\left(\Sigma_{+}^{\infty} X\right)^{S_{\bullet}^{1}} \wedge\left(\Sigma_{+}^{\infty} X\right)^{S_{\bullet}^{1}}\right) \simeq \operatorname{Tot}\left(\left(\Sigma_{+}^{\infty} X\right)^{S_{\bullet}^{1}}\right) \wedge \operatorname{Tot}\left(\left(\Sigma_{+}^{\infty} X\right)^{S_{\bullet}^{1}}\right) .
$$

By the above, both sides are weakly equivalent to $\Sigma_{+}^{\infty} \mathcal{L} X \wedge \Sigma_{+}^{\infty} \mathcal{L} X$.

Remark 5.3. The totalization of $\left(\Sigma_{+}^{\infty} X\right)^{S_{\bullet}^{1}}$ as a coalgebra spectrum (in the infinity categorical framework) agrees with the totalization as a spectrum. For this, we observe that by the above lemma, the totalization in spectra is already a coalgebra spectrum, and its coalgebra structure maps come from the structure maps on the cosimplicial coalgebra spectrum $\Sigma_{+}^{\infty} X^{S_{\bullet}^{1}}$. Hence the totalization in spectra $\operatorname{Tot}\left(\Sigma_{+}^{\infty} X^{S_{\bullet}^{1}}\right)$ already satisfies the universal property of the totalization in coalgebra spectra and the natural map

$$
\operatorname{Tot}_{\mathrm{CoCAlg}}\left(\left(\Sigma_{+}^{\infty} X\right)^{S_{\bullet}^{1}}\right) \rightarrow \operatorname{Tot}_{\mathrm{Spec}}\left(\left(\Sigma_{+}^{\infty} X\right)^{S_{\bullet}^{1}}\right)
$$

must be an equivalence.

The result of Lemma 5.1 is analogous to the result for topological Hochschild homology that when $R$ is commutative, $\operatorname{THH}(R)$ is an algebra spectrum. Further, for commutative $R$, THH $(R)$ is known to be a Hopf algebra over $R$ in the homotopy category $[16,29]$. Below, we consider the analogous result for the topological coHochschild homology of suspension spectra. Our proof relies on the identification of the coalgebra structure on $\operatorname{coTHH}\left(\Sigma_{+}^{\infty} X\right)$ from Lemma 5.1 and in particular on the consequence of this structure noted in Remark 5.3. This allows us to work in the context of cocommutative coalgebra spectra and we take advantage of additional formal properties available there.

Proposition 5.4. For a simply connected space $X$, $\operatorname{coTHH}\left(\Sigma_{+}^{\infty} X\right)$ is a $\square_{\Sigma_{+}^{\infty} X^{-}}$ coalgebra; that is, $\operatorname{coTHH}\left(\Sigma_{+}^{\infty} X\right)$ defines an object in the $\infty$-category

$$
\mathrm{CoCAlg}\left(\mathrm{CoCAlg}(\mathrm{Spec}) / \Sigma_{+}^{\infty} X\right) \text {. }
$$

Proof. Observe that the inclusion of the basepoint $* \rightarrow S_{\bullet}^{1}$ induces a map of cosimplicial coalgebra spectra

$$
\operatorname{coTHH}^{\bullet}\left(\Sigma_{+}^{\infty} X\right) \rightarrow \Sigma_{+}^{\infty} X
$$

where the target is the constant cosimplicial coalgebra spectrum. Totalizing in $\mathrm{CoCAlg}(\mathrm{Spec})$ we obtain a map $\operatorname{coTHH}\left(\Sigma_{+}^{\infty} X\right) \rightarrow \Sigma_{+}^{\infty} X$ and by Lemma 5.1 , this is a map of cocommutative coalgebra spectra. Hence $\operatorname{coTHH}\left(\Sigma_{+}^{\infty} X\right)$ is an object of $\mathrm{CoCAlg}(\mathrm{Spec}) / \Sigma_{+}^{\infty} X$. Proposition 4.5 shows that $\operatorname{coTHH}\left(\Sigma_{+}^{\infty} X\right)$ is then a cocommutative $\square_{\Sigma_{+}^{\infty} X}$-coalgebra.

It is useful to be more explicit in identifying the comultiplication structure on $\operatorname{coTHH}\left(\Sigma_{+}^{\infty} X\right)$. Unwinding the proof of Proposition 4.5, the comultiplication over $\Sigma_{+}^{\infty} X$ on $\operatorname{coTHH}\left(\Sigma_{+}^{\infty} X\right)$ of Proposition 5.4 is a map of cocommutative coalgebra spectra

$$
\operatorname{coTHH}\left(\Sigma_{+}^{\infty} X\right) \rightarrow \operatorname{coTHH}\left(\Sigma_{+}^{\infty} X\right) \square_{\Sigma_{+}^{\infty} X} \operatorname{coTHH}\left(\Sigma_{+}^{\infty} X\right)
$$


where the target is the monoidal product in $\mathrm{CoCAlg}(\mathrm{Spec}) / \Sigma_{+}^{\infty} X$, defined by pullback over $\Sigma_{+}^{\infty} X$. Since $S_{\bullet}^{1} \vee S_{\bullet}^{1}$ is the pushout

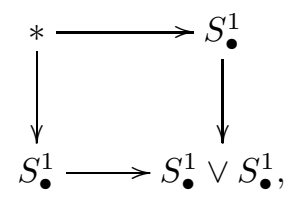

$\left(\Sigma_{+}^{\infty} X\right)^{S_{\bullet}^{1} \vee S_{\bullet}^{1}}$ is the pullback in cosimplicial coalgebra spectra



Totalization preserves pullbacks in $\infty$-categories, so we have a pullback diagram in cocommutative coalgebra spectra

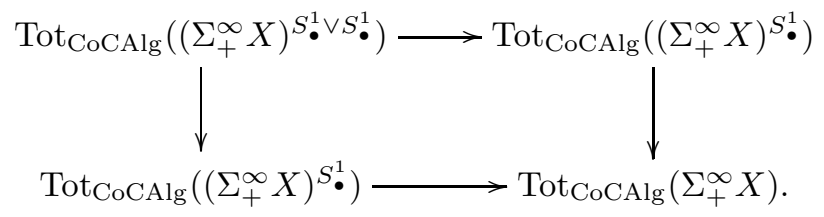

The totalization in spectra of $\left(\Sigma_{+}^{\infty} X\right)^{S_{\bullet}^{1}}$ and $\left(\Sigma_{+}^{\infty} X\right)^{*}$ are already cocommutative coalgebras, so we identify this pullback as

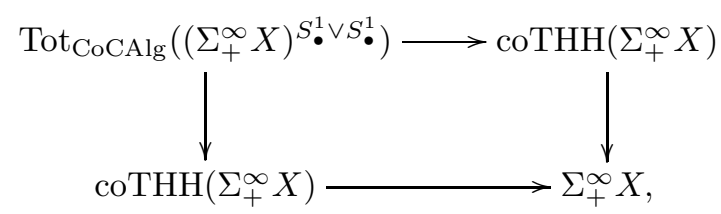

and thus we have made the identification

$$
\operatorname{coTHH}\left(\Sigma_{+}^{\infty} X\right) \square_{\Sigma_{+}^{\infty} X} \operatorname{coTHH}\left(\Sigma_{+}^{\infty} X\right)=\operatorname{Tot}_{\mathrm{CoCAlgSpec}}\left(\Sigma_{+}^{\infty} X\right)^{S_{\bullet}^{1} \vee S_{\bullet}^{1}} .
$$

The comultiplication map $\operatorname{coTHH}\left(\Sigma_{+}^{\infty} X\right) \rightarrow \operatorname{coTHH}\left(\Sigma_{+}^{\infty} X\right) \square_{\Sigma_{+}^{\infty} X} \operatorname{coTHH}\left(\Sigma_{+}^{\infty} X\right)$ is therefore the totalization in $\mathrm{CoCAlg}(\mathrm{Spec})$ of the map of cosimplicial coalgebra spectra

$$
\left(\Sigma_{+}^{\infty} X\right)^{S_{\bullet}^{1}} \rightarrow\left(\Sigma_{+}^{\infty} X\right)^{S_{\bullet}^{1} \vee S_{\bullet}^{1}}
$$

obtained by cotensoring with the fold map $S_{\bullet}^{1} \vee S_{\bullet}^{1} \rightarrow S_{\bullet}^{1}$ of simplicial sets.

The cocommutative coalgebra structure on $\operatorname{coTHH}\left(\Sigma_{+}^{\infty} X\right)$ is thus fairly formal in the sense that it does not use any special properties of $S_{\bullet}^{1}$. Showing that $\operatorname{coTHH}\left(\Sigma_{+}^{\infty} X\right)$ admits multiplication-like structure does require specific properties of the circle. In particular, as in Section 3, this requires using alternate simplicial models of the circle. We first show that $\operatorname{coTHH}\left(\Sigma_{+}^{\infty} X\right)$ can also be constructed using a double circle model of $S^{1}$.

Lemma 5.5. Let $\mathcal{C}$ be a Cartesian monoidal $\infty$-category. Recall the double circle $d S_{\bullet}^{1}=\left(\Delta^{1} \amalg \Delta^{1}\right) \amalg_{\left(\partial \Delta^{1} \amalg \partial \Delta^{1}\right)} \partial \Delta^{1}$ defined in Section 3, and let $\pi: d S_{\bullet}^{1} \rightarrow S_{\bullet}^{1}$ be 
the map of simplicial sets given by collapsing one of the 1-simplices. For an object $C \in \mathcal{C}$, totalization in $\mathcal{C}$ induces an equivalence

$$
\lim _{\Delta} C^{S_{\bullet}^{1}} \rightarrow \lim _{\Delta} C^{d S_{\bullet}^{1}}
$$

Proof. The collapse map $d S_{\bullet}^{1} \rightarrow S_{\bullet}^{1}$ is the induced map on pushouts of the diagrams

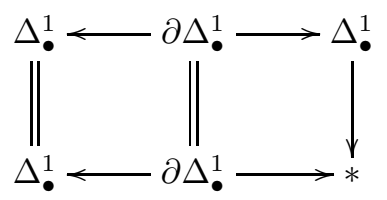

Hence the map $C^{S_{\bullet}^{1}} \rightarrow C^{d S^{1}}$ of cosimplicial objects of $\mathcal{C}$ is the induced map on the pullbacks below

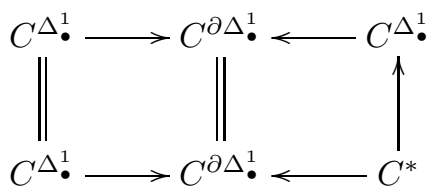

We are interested in the map $\lim _{\Delta} C^{S_{\bullet}^{1}} \rightarrow \lim _{\Delta} C^{d S_{\bullet}^{1}}$. Since limits commute, as follows from [32, Lemma 2.4.1], it is equivalent to calculate the pullback of the diagram of totalizations in $\mathcal{C}$ :

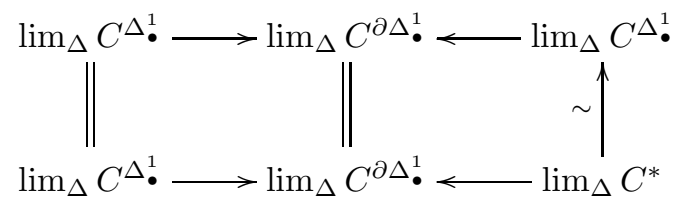

By Lemma 4.6, the right vertical map is an equivalence, and thus the pullbacks of these equivalent diagrams are equivalent.

Proposition 5.6. Let $X$ be a simply connected space. Then $\operatorname{coTHH}\left(\Sigma_{+}^{\infty} X\right)$ is a Hopf monoid in the homotopy category of coalgebra spectra over $\Sigma_{+}^{\infty} X$ : it is a bimonoid with an antipode map.

Intuitively, this structure should be thought of as that of a $\square_{\Sigma_{+}^{\infty} X}$-Hopf algebra structure on $\operatorname{coTHH}\left(\Sigma_{+}^{\infty} X\right)$ up to homotopy.

Proof. We must show that $\operatorname{coTHH}\left(\Sigma_{+}^{\infty} X\right)$ has the structure of a comonoid and a monoid and define the antipode map relating these structures. The comonoid structure is that of Proposition 5.4, with comultiplication

$$
\operatorname{coTHH}\left(\Sigma_{+}^{\infty} X\right) \rightarrow \operatorname{coTHH}\left(\Sigma_{+}^{\infty} X\right) \square_{\Sigma_{+}^{\infty} X} \operatorname{coTHH}\left(\Sigma_{+}^{\infty} X\right)
$$

identified as arising from the fold map $S_{\bullet}^{1} \vee S_{\bullet}^{1} \rightarrow S_{\bullet}^{1}$ of the simplicial circle and counit identified as arising from the inclusion of the basepoint $* \rightarrow S_{\bullet}^{1}$.

We next define the monoid structure on $\operatorname{coTHH}\left(\Sigma_{+}^{\infty} X\right)$, over $\Sigma_{+}^{\infty} X$. Recall that there is a based simplicial pinch map

$$
\psi: d S_{\bullet}^{1} \rightarrow S_{\bullet}^{1} \vee S_{\bullet}^{1},
$$


where $d S_{\bullet}^{1}$ is the double circle as above. This induces maps of cosimplicial coalgebra spectra

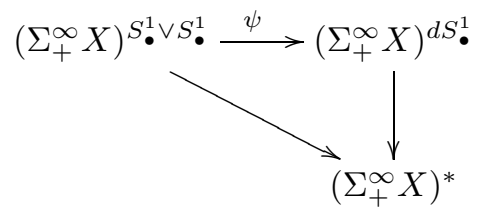

After totalization in $\mathrm{CoCAlg}(\mathrm{Spec})$ this yields a map over $\Sigma_{+}^{\infty} X$

$$
\psi: \operatorname{coTHH}\left(\Sigma_{+}^{\infty} X\right) \square_{\Sigma_{+}^{\infty} X} \operatorname{coTHH}\left(\Sigma_{+}^{\infty} X\right) \rightarrow \operatorname{Tot}_{\mathrm{CoCAlg}}\left(\left(\Sigma_{+}^{\infty} X\right)^{d S_{\bullet}^{1}}\right) .
$$

The collapse map $\pi: d S_{\bullet}^{1} \rightarrow S_{\bullet}^{1}$ that takes the second $\Delta_{\bullet}^{1}$ to the basepoint induces a map

$$
\pi: \operatorname{Tot}_{\mathrm{CoCAlg}}\left(\Sigma_{+}^{\infty} X\right)^{S_{\bullet}^{1}} \rightarrow \operatorname{Tot}_{\mathrm{CoCAlg}}\left(\Sigma_{+}^{\infty} X\right)^{d S_{\bullet}^{1}}
$$

which is an equivalence in $\mathrm{CoCAlg}$ (Spec) by Lemma 5.5 and thus in spectra. Thus, by Remark 5.3 , the target of $\psi$ is weakly equivalent to $\operatorname{coTHH}\left(\Sigma_{+}^{\infty} X\right)$, so we may view $\psi$ as the multiplication of a monoid structure. The unit map $\Sigma_{+}^{\infty} X \rightarrow$ $\operatorname{coTHH}\left(\Sigma_{+}^{\infty} X\right)$ is obtained similarly from the simplicial map $S_{\bullet}^{1} \rightarrow *$. The monoid axioms arise from suitably commuting diagrams or homotopy-commuting diagrams of simplicial sets, as in the proof of Proposition 3.8.

The antipode map $\chi: \operatorname{coTHH}\left(\Sigma_{+}^{\infty} X\right) \rightarrow \operatorname{coTHH}\left(\Sigma_{+}^{\infty} X\right)$ is defined using the double circle $d S_{\bullet}^{1}$. The double circle enjoys a simplicial flip map $\chi^{\prime}: d S_{\bullet}^{1} \rightarrow d S_{\bullet}^{1}$. Cotensoring with $\chi^{\prime}$ and using the equivalence $\pi$ produces the zigzag

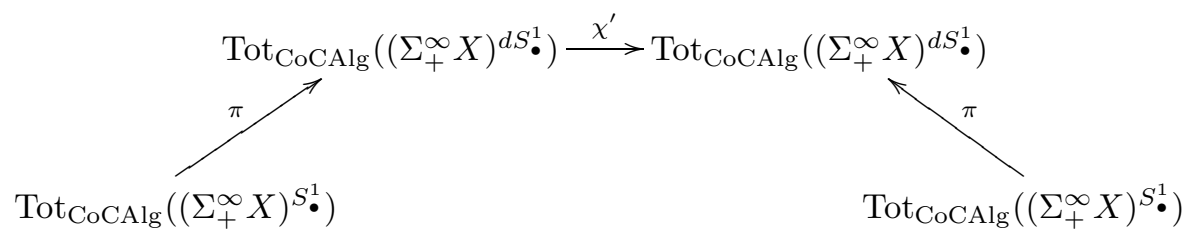

and hence an antipode map $\chi: \operatorname{coTHH}\left(\Sigma_{+}^{\infty} X\right) \rightarrow \operatorname{coTHH}\left(\Sigma_{+}^{\infty} X\right)$ in the homotopy category.

To check the compatibility diagram involving the antipode, we also need a triple model for the circle. As in [2], let $t S_{\bullet}^{1}$ denote $\partial \Delta^{2}$, with three non-degenerate 1simplices as shown:

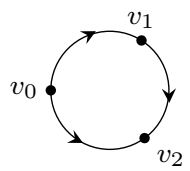

Let

$$
\psi_{1}: t S_{\bullet}^{1} \rightarrow S_{\bullet}^{1} \vee d S_{\bullet}^{1}
$$

be the simplicial map that identifies the $v_{0}$ and $v_{1}$ in $\partial \Delta^{2}$ and takes the face opposite $v_{0}$ to the first $\Delta^{1}$ in $d S^{1}$. Let

$$
\psi_{2}: t S_{\bullet}^{1} \rightarrow d S_{\bullet}^{1} \vee S_{\bullet}^{1}
$$

be the simplicial map that identifies the vertices $v_{1}$ and $v_{2}$ in $\partial \Delta^{2}$ and takes the face opposite $v_{2}$ to the first $\Delta^{1}$ in $d S^{1}$. Then the commutative diagram in Definition 2.13 
comes from cotensoring with the following homotopy commutative diagram of simplicial sets:

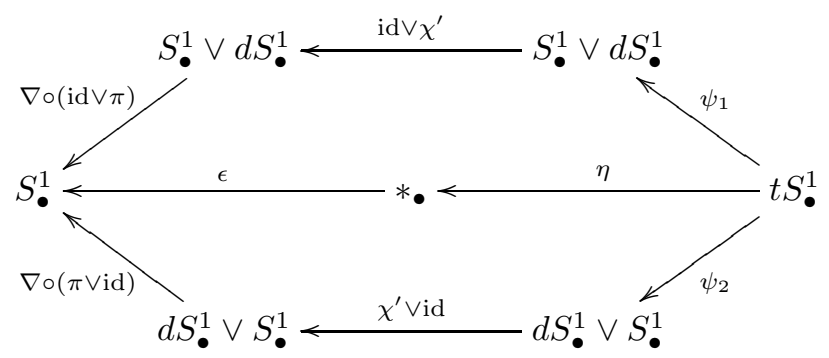

This diagram appears in the proof of [2, Theorem 3.9], where the authors discuss the homotopy commutativity. Note that the names of the maps $\epsilon$ and $\eta$ are reversed from their choice of names because these maps play opposite roles in the coalgebra and algebra cases. An argument analogous to that of Lemma 5.5 shows that cotensoring $\Sigma_{+}^{\infty} X$ with $t S_{\bullet}^{1}$ produces a coalgebra spectrum that is again equivalent to $\operatorname{coTHH}\left(\Sigma_{+}^{\infty} X\right)$.

Before looking at the implications of this result for the homology of free loop spaces, we prove the following lemma. Recall that a map of coalgebras $A \rightarrow C$ allows us to view $A$ as a right or left $C$-comodule, similar to Observation 2.8.

Lemma 5.7. Let $k$ be a field and let $A, B$ and $C$ be $H k$-coalgebras. Let $X$ be the homotopy pullback in $H k$-module spectra of the diagram



If $\pi_{*}(A)$ or $\pi_{*}(B)$ is coflat as a comodule over $\pi_{*}(C)$, then

$$
\pi_{*}(X) \cong \pi_{*}(A) \square_{\pi_{*}(C)} \pi_{*}(B)
$$

Proof. Consider the cosimplicial cobar spectrum $\Omega_{H k}^{\bullet}(A, C, B)$ with $n$th cosimplicial level

$$
A \wedge_{H k} C^{\wedge_{H k}(n)} \wedge_{H k} B
$$

as discussed, for example, in $[20, \S 3.3]$. Applying homotopy to this cobar spectrum produces a graded cobar complex; that is,

$$
\pi_{*}\left(\Omega_{H k}^{\bullet}(A, C, B)\right) \cong \Omega_{k}^{\bullet}\left(\pi_{*}(A), \pi_{*}(C), \pi_{*}(B)\right)
$$

with $n$th cosimplicial level

$$
\pi_{*}(A) \otimes_{k} \pi_{*}(C)^{\otimes_{k} n} \otimes_{k} \pi_{*}(B) .
$$

Consider the Bousfield-Kan spectral sequence associated to the cosimplicial spectrum $\Omega_{H k}^{\bullet}(A, C, B)$. To calculate the $E_{2}$-page, note that if we replace $A$ by $C$, then $\Omega_{k}^{\bullet}\left(\pi_{*}(C), \pi_{*}(C), \pi_{*}(B)\right)$ produces an injective resolution of $\pi_{*}(B)$ and applying the functor

$$
\pi_{*}(A) \square_{\pi_{*}(C)}-
$$

recovers $\Omega_{k}^{\bullet}\left(\pi_{*}(A), \pi_{*}(C), \pi_{*}(B)\right)$. Hence the $E_{2}$ page is given by

$$
\text { Cotor }_{\pi_{*}(C)}\left(\pi_{*}(A), \pi_{*}(B)\right) \text {. }
$$


If $\pi_{*}(A)$ or $\pi_{*}(B)$ is coflat over $\pi_{*}(C)$, then the spectral sequence collapses with

$$
\pi_{*}(A) \square_{\pi_{*}(C)} \pi_{*}(B)
$$

in the first column and zero everywhere else. This is the right-hand side above, so the proposition follows by showing that the spectral sequence converges to the left hand side. Since the spectral sequence collapses under the coflatness conditions, it converges completely to $\pi_{*} \operatorname{Tot} \Omega_{H k}^{\bullet}(A, C, B) \cong \pi_{*}(X)$ by [6, IX.5.4].

Proposition 5.6 then yields the following algebraic structure on the homology of free loop spaces.

Corollary 5.8. For $X$ a simply connected space and $k$ a field, if $H_{*}(\mathcal{L} X ; k)$ is coflat as a comodule over $H_{*}(X ; k)$, then $H_{*}(\mathcal{L} X ; k)$ is a $\square_{H_{*}(X ; k)}$-Hopf algebra.

Proof. Recall that for a simply connected space $X, \operatorname{coTHH}\left(\Sigma_{+}^{\infty} X\right) \simeq \Sigma_{+}^{\infty} \mathcal{L} X$. The corollary follows from Proposition 5.6 by applying homology once we identify

$$
H_{*}\left(\operatorname{coTHH}\left(\Sigma_{+}^{\infty} X\right) \square_{\Sigma_{+}^{\infty} X} \operatorname{coTHH}\left(\Sigma_{+}^{\infty} X\right) ; k\right)
$$

with

$$
H_{*}\left(\operatorname{coTHH}\left(\Sigma_{*}^{\infty} X\right) ; k\right) \square_{H_{*}(X ; k)} H_{*}\left(\operatorname{coTHH}\left(\Sigma_{+}^{\infty} X\right) ; k\right) .
$$

This identification follows from the stability of the $\infty$-category of coalgebra spectra. Stability implies that the homotopy pullback square in Diagram (1) is also a homotopy pushout square. After smashing with $H k$, we still have a homotopy pushout and, by stability, also a homotopy pullback. Passing to homotopy groups and applying Lemma 5.7 above then gives the identification.

We thus obtain a comultiplication map

$$
H_{*}\left(\operatorname{coTHH}\left(\Sigma_{+}^{\infty} X\right) ; k\right) \rightarrow H_{*}\left(\operatorname{coTHH}\left(\Sigma_{+}^{\infty} X\right) ; k\right) \square_{H_{*}(X ; k)} H_{*}\left(\operatorname{coTHH}\left(\Sigma_{+}^{\infty} X\right) ; k\right)
$$

by applying homology to the comultiplication map produced in Proposition 5.6, and likewise for the remaining structure maps of a $\square_{H_{*}(X ; k)}$-Hopf algebra.

Remark 5.9. One can show that the Hopf structure of Proposition 5.6 arises from suspending space level structure, in the following sense. As mentioned at the beginning of this section, Hess and Shipley [20, Appendix A] prove that for $X$ simply connected, $\operatorname{coTHH}\left(\Sigma_{+}^{\infty} X\right) \simeq \Sigma_{+}^{\infty}(\mathcal{L} X)$. The main tool is a convergence result due to Bousfield and the argument of [20, Appendix A] applies directly to show that for $X$ simply connected,

$$
\operatorname{Tot}_{\text {Spec }}\left(\Sigma_{+}^{\infty}\left(\operatorname{Map}\left(S_{\bullet}^{1} \vee S_{\bullet}^{1}, X\right)\right)\right) \simeq \Sigma_{+}^{\infty}\left(\operatorname{Tot}_{\operatorname{Top}} \operatorname{Map}\left(S_{\bullet}^{1} \vee S_{\bullet}^{1}, X\right)\right) .
$$

There is a standard identification of the right hand side as $\Sigma_{+}^{\infty}\left(\mathcal{L} X \times_{X} \mathcal{L} X\right)$, and the fiberwise diagonal and concatenation product of string topology give a Hopfmonoid structure for $\mathcal{L} X$ as a space over $X$. Since this structure arises from the same types of fold and diagonal maps on $S^{1} \vee S^{1}$ as appear in the structure from Proposition 5.6, we may identify the two structures provided that

$$
\operatorname{Tot}_{\text {Spec }}\left(\Sigma_{+}^{\infty}\left(\operatorname{Map}\left(S_{\bullet}^{1} \vee S_{\bullet}^{1}, X\right)\right)\right) \simeq \Sigma_{+}^{\infty} \mathcal{L} X \square_{\Sigma_{+}^{\infty} X} \Sigma_{+}^{\infty} \mathcal{L} X
$$

Because $\Sigma_{+}^{\infty}$ is a product-preserving functor from unbased spaces to cocommutative coalgebra spectra, we observe that $\Sigma_{+}^{\infty}\left(\operatorname{Map}\left(S_{\bullet}^{1} \vee S_{\bullet}^{1}, X\right)\right)$ is the underlying cosimplicial spectrum of the cosimplical coalgebra spectrum $\left(\Sigma_{+}^{\infty} X\right)^{S_{\bullet}^{1} \vee S_{\bullet}^{1}}$. The 
Hess-Shipley argument shows that the totalization in spectra is a suspension spectrum, and hence already a coalgebra spectrum, and therefore

$$
\operatorname{Tot}_{\mathrm{Spec}}\left(\Sigma_{+}^{\infty}\left(\operatorname{Map}\left(S_{\bullet}^{1} \vee S_{\bullet}^{1}, X\right)\right)\right) \simeq \operatorname{Tot}_{\mathrm{CoCAlg}}\left(\left(\Sigma_{+}^{\infty} X\right)^{S_{\bullet}^{1} \vee S_{\bullet}^{1}}\right) .
$$

Then the identification of Diagram (1) shows that the right hand side above is precisely $\Sigma_{+}^{\infty} \mathcal{L} X \square_{\Sigma_{+}^{\infty} X} \Sigma_{+}^{\infty} \mathcal{L} X$. This gives a more "geometric" description of this Hopf structure, but we require suitable cosimplicial descriptions in order to use this structure in the spectral sequence results of the next section.

\section{Hopf Structure in the coBökstedt Spectral Sequence}

An essential tool for computing topological Hochschild homology is the Bökstedt spectral sequence of [5]. In [4], we showed that there is an analogous coBökstedt spectral sequence for computing topological coHochschild homology. In this section, we show that this spectral sequence has additional algebraic structure, which we exploit in Section 7 to make free loop space computations. We first recall the structure and convergence results about the coBökstedt spectral sequence from [4].

Theorem 6.1 ([4]). Let $k$ be a field. Let $C$ be a coalgebra spectrum. The BousfieldKan spectral sequence for the cosimplicial spectrum coTHH $^{\bullet}(C)$ gives a coBökstedt spectral sequence for calculating $H_{t-s}(\operatorname{coTHH}(C) ; k)$ with $E_{2}$-page

$$
E_{2}^{s, t}=\operatorname{coHH}_{s, t}^{k}\left(H_{*}(C ; k)\right)
$$

given by the classical coHochschild homology of $H_{*}(C ; k)$.

We also have the following convergence results.

Proposition 6.2 ([4]). If for each $s$ there is an $r$ such that $E_{r}^{s, s+i}=E_{\infty}^{s, s+i}$, then the coBökstedt spectral sequence for $\operatorname{coTHH}(C)$ converges completely to

$$
\pi_{*} \operatorname{Tot}\left(\operatorname{coTHH}^{\bullet}(C) \wedge H k\right) .
$$

Remark 6.3. In [4], the authors use the simplicial model category structure on spectra to define the homotopy limits/totalizations and homotopy types used in defining $\operatorname{coTHH}(C)$. In this paper, we choose instead to use the $\infty$-categorical framework for these constructions, as is reflected in the omission of the (co)fibrancy conditions that appeared in the statements of these results in [4].

Note that the general construction of a map of the form $\operatorname{Hom}(X, Y) \wedge Z \rightarrow$ $\operatorname{Hom}(X, Y \wedge Z)$ yields a natural map

$$
P: \operatorname{Tot}\left(\operatorname{coTHH}^{\bullet}(C)\right) \wedge H k \rightarrow \operatorname{Tot}\left(\operatorname{coTHH}^{\bullet}(C) \wedge H k\right) .
$$

From [4], if this map $P$ is an isomorphism in homotopy, and the conditions on $E_{r}^{s, s+i}$ in Proposition 6.2 hold, then the coBökstedt spectral sequence for coTHH $(C)$ converges completely to $H_{*}(\operatorname{coTHH}(C) ; k)$.

Further, in [4] it is shown that the coBökstedt spectral sequence of Theorem 6.1 is a spectral sequence of coalgebras. Hence if $C$ is a connected cocommutative coalgebra, for each $r>1$ there is a comultiplication

$$
\psi: E_{r}^{* *} \rightarrow E_{r}^{* *} \otimes_{k} E_{r}^{* *},
$$

and the differentials $d_{r}$ respect the comultiplication. 
Based on algebraic structures in the classical Bökstedt spectral sequence, one might expect to have additional algebraic structure in the coBökstedt spectral sequence. In particular, in [2] Angeltveit and Rognes show that, under a flatness assumption, the Bökstedt spectral sequence

$$
E_{*, *}^{2}=\mathrm{HH}_{*}\left(H_{*}\left(R ; \mathbb{F}_{p}\right)\right) \Rightarrow H_{*}\left(\mathrm{THH}(R) ; \mathbb{F}_{p}\right)
$$

is a spectral sequence of Hopf algebras over $H_{*}\left(R ; \mathbb{F}_{p}\right)$. In this section we consider what the analogous algebraic structure is on the coBökstedt spectral sequence. Under coflatness conditions, we prove that the coBökstedt spectral sequence is a spectral sequence of $\square_{H_{*}(C ; k)}$-Hopf algebras, in the sense of the following definitions.

Definition 6.4. Let $D$ be a cocommutative coalgebra over a field $k$. A differential bigraded $\square_{D}$-algebra $\left(E^{*, *}, d\right)$ is a bigraded $D$-bicomodule $E^{*, *}$, a map of $D$-bicomodules

$$
d: \bigoplus_{q-p=n} E^{p, q} \rightarrow \bigoplus_{s-r=n-1} E^{r, s}
$$

and a $\square_{D}$-multiplication structure

$$
\mu: E^{s, t} \square_{D} E^{u, v} \rightarrow E^{s+u, t+v}
$$

with a unit $\eta: D \rightarrow E^{*, *}$, such that the usual associativity and unitality diagrams commute. The differential is compatible with the product, in the sense that $d$ must satisfy the Leibniz rule:

$$
d \circ \mu=\mu \circ\left(d \square_{D} \mathrm{id}+(-1)^{s+t} \mathrm{id} \square_{D} d\right) .
$$

Definition 6.5. Let $D$ be a cocommutative coalgebra over a field $k$. We say that a spectral sequence $\left\{E_{r}, d_{r}\right\}$ is a spectral sequence of $\square_{D}$-algebras if every $D$-comodule $E_{r}^{s, t}$ is coflat, and for every $r \geq 1,\left(E_{r}^{*, *}, d_{r}\right)$ is a differential bigraded $\square_{D}$-algebra, with multiplication $\mu_{r}$, and if the multiplication $\mu_{r+1}$ is the composite

$$
\begin{aligned}
\mu_{r+1}: E_{r+1} \square_{D} E_{r+1} \cong H_{*}\left(E_{r} ; k\right) & \square_{D} H_{*}\left(E_{r} ; k\right) \\
& \cong H_{*}\left(E_{r} \square_{D} E_{r} ; k\right) \stackrel{H_{*}\left(\mu_{r}\right)}{\longrightarrow} H_{*}\left(E_{r} ; k\right) \cong E_{r+1} .
\end{aligned}
$$

Here the isomorphism $H_{*}\left(E_{r} ; k\right) \square_{D} H_{*}\left(E_{r} ; k\right) \cong H_{*}\left(E_{r} \square_{D} E_{r} ; k\right)$ is the Künneth isomorphism for $\square_{D}$, as in Proposition 3.4. This uses the hypothesis that $E_{r}^{s, t}$ is coflat.

We now also define the notion of a spectral sequence of $\square_{D}$-coalgebras.

Definition 6.6. Let $D$ be a cocommutative coalgebra over a field $k$. A differential

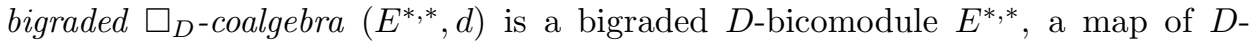
bicomodules

$$
d: \bigoplus_{q-p=n} E^{p, q} \rightarrow \bigoplus_{s-r=n-1} E^{r, s},
$$

and a $\square_{D}$-comultiplication structure

$$
\triangle: E^{s, t} \rightarrow \bigoplus_{\substack{u+w=s \\ v+x=t}} E^{u, v} \square_{D} E^{w, x}
$$


with a counit $\epsilon: E^{*, *} \rightarrow D$, such that the usual coassociativity and counitality diagrams commute. The differential is compatible with the comultiplication, in the sense that $d$ must satisfy the coLeibniz rule:

$$
\triangle \circ d=\left(d \square \mathrm{id}+(-1)^{u+v} \mathrm{id} \square d\right) \circ \triangle .
$$

Definition 6.7. Let $D$ be a cocommutative coalgebra over a field $k$. We say that a spectral sequence $\left\{E_{r}, d_{r}\right\}$ is a spectral sequence of $\square_{D}$-coalgebras if for every $r \geq 1$, $\left(E_{r}^{*, *}, d_{r}\right)$ is a differential bigraded $\square_{D}$-coalgebra, with comultiplication $\triangle_{r}$, and if the comultiplication $\triangle_{r+1}$ is the composite

$$
\begin{aligned}
\triangle_{r+1}: E_{r+1} \cong H_{*}\left(E_{r} ; k\right) \stackrel{H_{*}\left(\triangle_{r}\right)}{\longrightarrow} & H_{*}\left(E_{r} \square_{D} E_{r} ; k\right) \\
\stackrel{\phi}{\longrightarrow} & H_{*}\left(E_{r} ; k\right) \square_{D} H_{*}\left(E_{r} ; k\right) \cong E_{r+1} \square_{D} E_{r+1} .
\end{aligned}
$$

Here the map $\phi$ is the map from the homology of the cotensor to the cotensor of the homologies, as in Proposition 3.4.

We now characterize a $\square$-Hopf algebra structure on a spectral sequence.

Definition 6.8. Let $D$ be a cocommutative coalgebra over a field $k$. A differential bigraded $\square_{D}$-Hopf algebra is a pair $\left(E^{*, *}, d\right)$ with the structure of both a differential bigraded $\square_{D}$-algebra and a differential bigraded $\square_{D}$-coalgebra. The multiplication, unit, comultiplication and counit must be compatible, as in Definition 2.12. Further, a differential bigraded $\square_{D}$-Hopf algebra must have differential $D$-bicomodule maps

$$
\chi: E^{s, t} \rightarrow E^{s, t}
$$

satisfying the commutative diagram of Definition 2.13 .

Definition 6.9. Let $D$ be a cocommutative coalgebra over a field $k$. We say that a spectral sequence $\left\{E_{r}, d_{r}\right\}$ is a spectral sequence of $\square_{D}$-Hopf algebras if for every $r \geq$ $1,\left(E_{r}^{*, *}, d_{r}\right)$ is a differential bigraded $\square_{D}$-Hopf algebra with multiplication $\mu_{r}$ and comultiplication $\triangle_{r}$, such that $\left\{E_{r}, d_{r}\right\}$ is a spectral sequence of $\square_{D}$-coalgebras and a spectral sequence of $\square_{D}$-algebras using this comultiplication and multiplication. Further, the map

$$
\chi_{r+1}: E_{r+1}^{s, t} \rightarrow E_{r+1}^{s, t}
$$

must be the induced map

$$
\chi_{r+1}=H_{*}\left(\chi_{r}\right): H_{*}\left(E_{r}^{s, t} ; k\right) \rightarrow H_{*}\left(E_{r}^{s, t} ; k\right) .
$$

Having established these definitions, we now consider the algebraic structure on the coBökstedt spectral sequence. We first prove that it is a spectral sequence of $\square$-coalgebras.

Theorem 6.10. Let $C$ be a connected cocommutative coalgebra spectrum, and let $k$ be a field. Then the coBökstedt spectral sequence is a spectral sequence of $\square_{H_{*}(C ; k)^{-}}$ coalgebras.

Proof. By definition, the coBökstedt spectral sequence for $C$ is the Bousfield-Kan spectral sequence for the cosimplicial object $\operatorname{coTHH}^{\bullet}(C) \wedge H k$, which we will denote $X^{\bullet}$. 
We consider the following commutative diagram of simplicial sets



where $\nabla$ and $\nabla^{\prime}$ are both simplicial fold maps. Note that we may identify $S_{\bullet}^{1} \vee S_{\bullet}^{1}$ with the coequalizer of the two maps $S_{\bullet}^{1} \amalg * \amalg S_{\bullet}^{1} \rightrightarrows S_{\bullet}^{1} \amalg S_{\bullet}^{1}$ that send the point * to the basepoint in either copy of $S_{\bullet}^{1}$.

On cosimplicial cotensors, and after smashing with $H k$, the diagram above yields a diagram of cosimplicial spectra

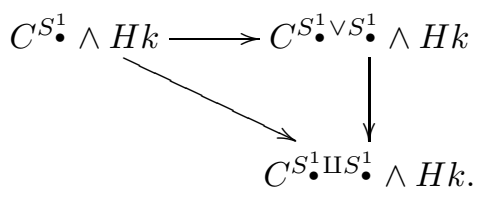

Furthermore the right vertical map here equalizes the two induced maps

$$
C^{S_{\bullet}^{1} \amalg S_{\bullet}^{1}} \wedge H k \rightrightarrows C^{S_{\bullet}^{1} \amalg * \amalg S_{\bullet}^{1}} \wedge H k .
$$

These maps of cosimplicial spectra induce maps of spectral sequences on the corresponding Bousfield-Kan spectral sequences: let $E_{r}^{*, *}$ denote the BousfieldKan spectral sequence for $X^{\bullet}$ (that is, the coBökstedt spectral sequence), let $D_{r}^{* \text {,* }}$ denote the spectral sequence for $C^{S_{\bullet}^{1} \vee S_{\bullet}^{1}} \wedge H k$, let ${ }^{\prime} D_{r}^{*, *}$ denote the spectral sequence for $C^{S_{\bullet}^{1} \amalg S_{\bullet}^{1}} \wedge H k$ and let ${ }^{\prime \prime} D_{r}^{*, *}$ denote the spectral sequence for $C^{S_{\bullet}^{1} \amalg * \amalg S_{\bullet}^{1}} \wedge H k$. In this notation, we have maps of spectral sequences

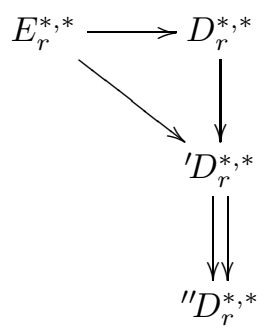

where the map $D_{r}^{*, *} \rightarrow{ }^{\prime} D_{r}^{*, *}$ equalizes the two lower vertical maps.

It is proved in [4] that the Alexander-Whitney map identifies the spectral sequence ' $D_{r}^{*, *}$ with the tensor product spectral sequence $E_{r}^{*, *} \otimes_{k} E_{r}^{*, *}$ from the $E_{2^{-}}$ page on, using Bousfield and Kan's work on pairings in Bousfield-Kan spectral sequences $[8,7]$. In fact, the identifications of Bousfield and Kan produce natural maps of spectral sequences

$$
{ }^{\prime} D_{r}^{*, *} \rightarrow E_{r}^{*, *} \otimes_{k} E_{r}^{*, *} \quad \text { and } \quad{ }^{\prime \prime} D_{r}^{*, *} \rightarrow E_{r}^{*, *} \otimes H_{*}(C) \otimes E_{r}^{*, *} .
$$

On the 1-pages, the maps in this direction are given by the shuffle map: this is the quasi-inverse of the map AW: $E_{1}^{*, *} \otimes_{k} E_{1}^{*, *} \rightarrow{ }^{\prime} D_{1}^{*, *}$ used in [4]. 
Thus, on the 1-pages, we have an induced map

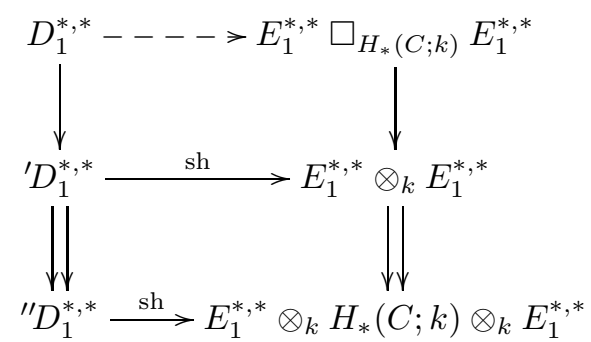

where sh denotes the shuffle map of the Eilenberg-Zilber theorem. Hence the composite of the spectral sequence map $E_{1}^{*, *} \rightarrow D_{1}^{*, *}$ with the dashed arrow above

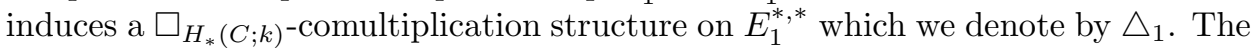
comultiplication $\triangle_{1}$ satisfies the coLeibniz rule because the composite

$$
E_{1}^{*, *} \rightarrow D_{1}^{*, *} \stackrel{\mathrm{sh}}{\longrightarrow} E_{1}^{*, *} \otimes_{k} E_{1}^{*, *}
$$

satisfies the coLeibniz rule and the differential $d_{r} \square 1 \pm 1 \square d_{r}$ on $E_{r}^{*, *} \square_{H_{*}(C ; k)} E_{r}^{*, *}$ is the restriction of the differential $d_{r} \otimes 1 \pm 1 \otimes d_{r}$ to the elements of $E_{r}^{*, *} \otimes_{k} E_{r}^{*, *}$ that are equalized.

The comultiplication on the $E_{2}$-page is induced similarly. By the usual calculation of the 1-page of a Bousfield-Kan spectral sequence, we make the identifications

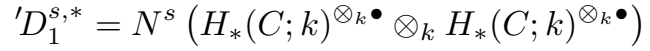

$$
\begin{aligned}
& { }^{\prime \prime} D_{1}^{s, *}=N^{s}\left(H_{*}(C ; k)^{\otimes_{k} \bullet} \otimes_{k} H_{*}(C ; k) \otimes_{k} H_{*}(C ; k)^{\otimes_{k} \bullet}\right) .
\end{aligned}
$$

Using these identifications, and the fact that normalization commutes with equalizers, we see that the $D_{1}$-page is

$$
D_{1}^{s, *}=N^{s}\left(H_{*}(C ; k)^{\otimes_{k} \bullet} \square_{H_{*}(C ; k)} H_{*}(C ; k)^{\otimes_{k} \bullet}\right) .
$$

The horizontal shuffle maps sh from the Eilenberg-Zilber map above induce isomorphisms on homology, so we have a diagram of maps of bigraded $k$-modules

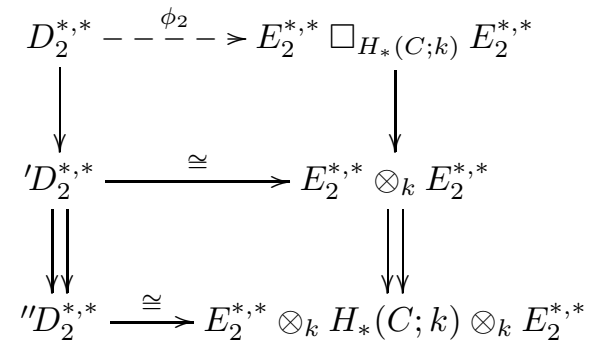

The induced dotted map is the map in the $\square_{H_{*}(C ; k)}$-Künneth Theorem, Proposition 3.4. We may thus define the comultiplication $\triangle_{2}$ on $E_{2}$ to be the composite of the map $E_{2}^{*, *} \rightarrow D_{2}^{*, *}$ and this induced dotted map; this clearly satisfies the condition of Definition 6.7. Again, since the middle map is a map of spectral sequences and the differential on $E_{2}^{*, *} \square_{H_{*}(C ; k)} E_{2}^{*, *}$ is restricted from that on $E_{2}^{*, *} \otimes_{k} E_{2}^{*, *}$, this comultiplication also satisfies the coLeibniz rule.

Because the middle and lower horizontal maps above are maps of spectral sequences, repeated application of the Künneth theorem for $k$-modules gives a similar diagram on the $r$-pages for $r \geq 2$; the Künneth theorem for $\square_{H_{*}(C ; k) \text {-comodules }}$ then induces the desired comultiplications $\triangle_{r}$ at each level. 
Remark 6.11. In the proof of Theorem 6.10, we use Bousfield and Kan's shuffle pairing ${ }^{\prime} D_{1}^{*, *} \rightarrow E_{1}^{*, *} \otimes_{k} E_{1}^{*, *}$, which - as remarked - is not the comparison map AW between these spectral sequences used in defining the coalgebra structure in [4]. Instead, the map AW is the quasi-inverse to Bousfield and Kan's pairing, which is constructed from the Künneth isomorphism and shuffle/Alexander-Whitney maps. This requires working over a field. Since the comparison map in Theorem 6.10 and the one of [4] are quasi-inverses at the $E_{1}$-page, we conclude that the $\square_{H_{*}(C ; k)^{-}}$ coalgebra structure on $E_{r}^{*, *}$ of Theorem 6.10 is the restriction of the $k$-coalgebra structure produced in [4].

Simplicial maps of the circle also induce the product structure on the coBökstedt spectral sequence. As in Section 3, we must use a double circle model of $S_{\bullet}^{1}$ to get a pinch map that is indeed simplicial. Recall the double circle model $d S_{\bullet}^{1}$ defined earlier:

$$
d S_{\bullet}^{1}=\left(\Delta^{1} \amalg \Delta^{1}\right) \amalg_{\left(\partial \Delta^{1} \amalg \partial \Delta^{1}\right)} \partial \Delta^{1} .
$$

There is a simplicial pinch map

$$
\psi: d S_{\bullet}^{1} \rightarrow S_{\bullet}^{1} \vee S_{\bullet}^{1}
$$

that collapses $\partial \Delta^{1}$ to a point. For $D$ a $k$-coalgebra, let $d \operatorname{coHH}^{\bullet}(D)$ denote the cosimplicial $k$-coalgebra $D^{d S_{\bullet}^{1}}$. To parallel Definition 3.1, we let $d \operatorname{coHH}_{*}(D)$ denote the homology of the chain complex (of $k$-modules) $C^{*}\left(d \mathrm{coHH}^{\bullet}(D)\right)$ associated to $d \mathrm{coHH}^{\bullet}(D)$ under the Dold-Kan correspondence. We will need the following lemma comparing coHochschild homology defined with the standard simplicial model of the circle to that defined with the double circle model. Recall that $\pi: d S_{\bullet}^{1} \rightarrow S_{\bullet}^{1}$ is the collapse map that takes the second $\Delta^{1}$ in $d S_{\bullet}^{1}$ to the basepoint. This lemma is the purely algebraic version of Lemma 5.5.

Lemma 6.12. Let $D$ be a cocommutative (graded) $k$-coalgebra. The map $\pi: d S_{\bullet}^{1} \rightarrow$ $S_{\bullet}^{1}$ induces an isomorphism of (bi-)graded abelian groups.

$$
\pi: \operatorname{coHH}_{*}(D) \rightarrow d \mathrm{coHH}_{*}(D)
$$

Proof. Following the proof of Lemma 5.5, we may take cotensors with the defining pushout diagrams of simplicial sets to model $\pi$ as the following map on pullbacks in cocommutative $k$-coalgebras:

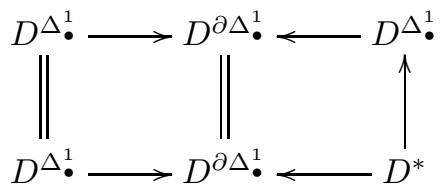

As in Observation 2.8, pullback in the category of cocommutative $k$-coalgebras agrees with the definition of $\square$-product. The coalgebra $D^{\partial \Delta_{\bullet}^{1}}$ is $D \otimes D$; this allows us to identify $\pi$ at the cosimplicial level with the map

$$
D^{\otimes \bullet+2} \square_{D \otimes D} D \rightarrow D^{\otimes \bullet+2} \square_{D \otimes D} D^{\otimes \bullet+2} .
$$

The map $\pi: \operatorname{coHH}_{*}(D) \rightarrow d \mathrm{coHH}_{*}(D)$ is the map on homology induced by the associated map of cochain complexes

$$
C^{*}\left(D^{\otimes \bullet+2} \square_{D \otimes D} D\right) \rightarrow C^{*}\left(D^{\otimes \bullet+2} \square_{D \otimes D} D^{\otimes \bullet+2}\right) .
$$


Observe that for each $n, D^{\otimes n+2}$ is a $D \otimes D$-comodule and by the Eilenberg-Zilber theorem for $D \otimes D$-comodules, this map is quasi-isomorphic to the map

$$
C^{*}\left(D^{\otimes \bullet+2}\right) \square_{D \otimes D} C^{*}(D) \rightarrow C^{*}\left(D^{\otimes \bullet+2}\right) \square_{D \otimes D} C^{*}\left(D^{\otimes \bullet+2}\right) .
$$

Now $C^{*}\left(D^{\otimes \bullet+2}\right)$ is an injective resolution of $D$ as a $D \otimes D$-bicomodule; the map $D \rightarrow C^{*}\left(D^{\Delta_{\bullet}^{1}}\right)$ is the augmentation of this resolution. By [15, Proposition 4.1] (and also see the discussion at the beginning of $\S 5$ there), this map induces an isomorphism on homology as desired.

Remark 6.13. Observe that the double circle $d S^{1}$ used in this section is a different version of the double circle than the model $d^{\prime} S^{1}$ used in Section 3. In particular, the orientation of one of the 1-simplices is reversed. In this section we use the model $d S^{1}$ so that the flip map will be simplicial. We choose to use the model $d^{\prime} S^{1}$ in Section 3 because it simplifies the chain-level formulas. This follows the choices made in [2] in the dual case.

Theorem 6.14. Let $C$ be a connected cocommutative coalgebra spectrum. If for $r \geq 2$, each $E_{r}^{*, *}(C)$ is coflat over $H_{*}(C ; k)$, then the coBökstedt spectral sequence is a spectral sequence of $\square_{H_{*}(C ; k)}$-Hopf algebras.

Proof. The comultiplication is given by Theorem 6.10 above. To obtain the multiplication, we use the double circle simplicial model of $S^{1}$ denoted $d S_{\bullet}^{1}$. Let $d E_{r}^{*, *}$ denote the Bousfield-Kan spectral sequence for the cosimplicial object $C^{d S^{1}} \wedge H k$. There are natural maps

$$
E_{r}^{*, *} \square_{H_{*}(C ; k)} E_{r}^{*, *} \stackrel{\phi_{r}}{\longleftarrow} D_{r}^{*, *} \stackrel{\psi}{\longrightarrow} d E_{r}^{*, *} \stackrel{\pi}{\longleftarrow} E_{r}^{*, *} .
$$

The map $\phi_{r}$ is the comparison map defined in the proof of Theorem 6.10 above. The map $\psi$ is induced by the simplicial pinch map $\psi: d S_{\bullet}^{1} \rightarrow S_{\bullet}^{1} \vee S_{\bullet}^{1}$, and the map $\pi$ is induced by the weak equivalence $d S_{\bullet}^{1} \rightarrow S_{\bullet}^{1}$ given by collapsing. By Lemma 6.12 the map $\pi$ is an isomorphism when $r \geq 2$.

We use induction to show that the map $\phi_{r}$ is an isomorphism for $r \geq 2$. We first establish the isomorphism when $r=2$. As in Theorem 6.10 above, the $D_{1}$-page can be identified as

$$
D_{1}^{s, *}=N^{s}\left(H_{*}(C ; k)^{\otimes_{k} \bullet} \square_{H_{*}(C ; k)} H_{*}(C ; k)^{\otimes_{k} \bullet}\right) \cong E_{1}^{s, *} \square_{H_{*}(C ; k)} E_{1}^{s, *},
$$

because the normalization of a cosimplicial object commutes with equalizers, as both are given at each level by limit constructions.

On the 1-page the map $\phi_{1}$ above is the shuffle map

$$
E_{1}^{s, *} \square_{H_{*}(C ; k)} E_{1}^{s, *} \rightarrow\left[E_{1}^{*, *} \square_{H_{*}(C ; k)} E_{1}^{*, *}\right]_{s, *} .
$$

which by Proposition 3.3 induces an isomorphism on homology:

$$
H_{*}\left(D_{1}^{*, *} ; k\right) \rightarrow H_{*}\left(E_{1}^{*, *} \square_{H_{*}(C ; k)} E_{1}^{*, *} ; k\right) .
$$

The left hand side is $D_{2}^{*, *}$. To calculate the right hand side, we can replace $E_{1}^{*, *}$, the normalized chain complex $N^{*}\left(H_{*}(C ; k)^{\otimes_{k} \bullet}\right)$, by the quasi-isomorphic unnormalized complex $H_{*}(C ; k)^{\otimes *}$, which is coflat over $H_{*}(C ; k)$. Since $E_{2}^{*, *}=H_{*}\left(E_{1}^{*, *} ; k\right)$ is coflat over $H_{*}(C ; k)$ by hypothesis, Proposition 3.4 implies the right hand side is

$$
H_{*}\left(E_{1}^{*, *} ; k\right) \square_{H_{*}(C ; k)} H_{*}\left(E_{1}^{*, *} ; k\right),
$$

and hence $\phi_{2}=H_{*}\left(\phi_{1}\right)$ is an isomorphism

$$
D_{2}^{*, *} \rightarrow E_{2}^{*, *} \square_{H_{*}(C ; k)} E_{2}^{*, *} .
$$


Now assume that $\phi_{r}$ is an isomorphism for some fixed $r \geq 2$. The isomorphism

$$
D_{r}^{*, *} \stackrel{\phi_{r}}{\longrightarrow} E_{r}^{*, *} \square_{H_{*}(C ; k)} E_{r}^{*, *}
$$

induces an isomorphism on homology. By hypothesis, $E_{r+1}^{*, *}$ is coflat over $H_{*}(C ; k)$, so Proposition 3.4 applies and the map $\phi_{r+1}=H_{*}\left(\phi_{r}\right)$ is an isomorphism

$$
D_{r+1}^{*, *} \stackrel{\cong}{\longrightarrow} E_{r+1}^{*, *} \square_{H_{*}(C ; k)} E_{r+1}^{*, *} .
$$

Thus by induction the map $\phi_{r}$ is an isomorphism for all $r \geq 2$.

The multiplication

$$
E_{r}^{*, *} \square_{H_{*}(C ; k)} E_{r}^{*, *} \stackrel{\mu_{r}}{\longrightarrow} E_{r}^{*, *}
$$

is then given by $\mu_{r}=\pi^{-1} \psi \phi_{r}^{-1}$. Since the maps $\phi_{r}$ constructed in the proof of Theorem 6.10 satisfy the coLeibniz rule and the remaining maps used in constructing $\mu_{r}$ are maps of spectral sequences, $\mu_{r}$ satisfies the Leibniz rule.

Finally, we define the antipode map $\chi_{r}: E_{r}^{*, *} \rightarrow E_{r}^{*, *}$ to be the map of spectral sequences

$$
\chi: E_{r}^{*, *} \stackrel{\pi}{\longrightarrow} d E_{r}^{*, *} \stackrel{\chi^{\prime}}{\longrightarrow} d E_{r}^{*, *} \stackrel{\pi^{-1}}{\longrightarrow} E_{r}^{*, *} .
$$

The middle map $\chi^{\prime}$ is the map of spectral sequences induced by the flip map on the double circle and the proof that this satisfies the required conditions for an antipode is analogous to the proof of Proposition 5.6. Again, we use that $\pi$ is an isomorphism for $r \geq 2$.

\section{Computational Results}

As discussed in Section 5, when $X$ is a simply connected space, $\operatorname{coTHH}\left(\Sigma_{+}^{\infty} X\right)$ can be identified with the suspension spectrum of the free loop space on $X$,

$$
\Sigma_{+}^{\infty} \mathcal{L} X \stackrel{\simeq}{\rightarrow} \operatorname{coTHH}\left(\Sigma_{+}^{\infty} X\right) .
$$

The coBökstedt spectral sequence thus provides a method for computing the homology of the free loop space [4, Corollary 4.5].

Proposition $7.1([4])$. Let $X$ be a simply connected space. If for each $s$ there is an $r$ such that $E_{r}^{s, s+i}=E_{\infty}^{s, s+i}$, the coBökstedt spectral sequence arising from the coalgebra $\Sigma_{+}^{\infty} X$ converges completely to

$$
H_{*}\left(\operatorname{coTHH}\left(\Sigma_{+}^{\infty} X\right) ; k\right) \cong H_{*}(\mathcal{L} X ; k) .
$$

In [4] the authors use the coBökstedt spectral sequence to compute the homology of free loop spaces $H_{*}(\mathcal{L} X ; k)$ for instance when $X$ is $\mathbb{C} P^{\infty}, B U(n), B S U(n), B S p(n)$, or products of these. These calculations use the $k$-coalgebra structure on the coBökstedt spectral sequence.

In this section we use the $\square$-Hopf algebra structure on the coBökstedt spectral sequence that we produced in Section 6 to carry out further computations of the homology of free loop spaces. In particular, we consider spaces with exterior cohomology. The (co)homology of free loop spaces of simply connected spaces with mod $p$ exterior cohomology has been considered, for instance, in [25], [23], [33], and [24]. Our approach yields new results, as consequences of the following general collapse result for the coBökstedt spectral sequence, which we prove later in this section. 
Theorem 7.2. Let $k$ be a field of characteristic $p$ and let $C$ be a cocommutative coalgebra spectrum whose homology coalgebra is

$$
H_{*}(C ; k)=\Lambda_{k}\left(y_{i_{1}}, y_{i_{2}}, \ldots, y_{i_{n}}\right) .
$$

Here the $y_{i_{j}}$ are cogenerators in odd degrees, $\left|y_{i_{j}}\right|=i_{j}$, and $i_{j+1} \geq i_{j} \geq 3$. Then if $\frac{i_{n}-2+\sum_{j=1}^{n} i_{j}}{i_{1}-1}<p$, the coBökstedt spectral sequence for $\operatorname{coTHH}(C)$ collapses at $E_{2}$, and

$$
E_{2} \cong E_{\infty} \cong \Lambda_{k}\left(y_{i_{1}}, y_{i_{2}}, \ldots, y_{i_{n}}\right) \otimes k\left[w_{i_{1}}, w_{i_{2}}, \ldots, w_{i_{n}}\right],
$$

with $y_{i_{j}}$ in bidegree $\left(0, i_{j}\right)$ and $w_{i_{j}}$ in bidegree $\left(1, i_{j}\right)$.

Letting $C=\Sigma_{+}^{\infty} X$, for $X$ a simply connected space with exterior cohomology, Theorem 7.2 yields results on the homology of free loop spaces. We first consider spaces $X$ whose cohomology is exterior on two generators. This case has been considered previously in work of Kuribayashi and Yamaguchi [25], and we compare our results to that previous work in Remark 7.4. The following result follows directly from Theorem 7.2.

Theorem 7.3. Let $k$ be a field of characteristic $p$ and let $X$ be a simply connected space whose cohomology is exterior on two generators in odd degrees,

$$
H^{*}(X ; k) \cong \Lambda_{k}\left(x_{i_{1}}, x_{i_{2}}\right),
$$

$\left|x_{i_{j}}\right|=i_{j}$, and $i_{1} \leq i_{2} \leq \frac{p-1}{2} i_{1}-\frac{p-1}{2}$. Then the homology of the free loop space on $X$ is given as a graded $k$-module by

$$
H_{*}(\mathcal{L} X ; k) \cong \Lambda_{k}\left(y_{i_{1}}, y_{i_{2}}\right) \otimes k\left[w_{i_{1}}, w_{i_{2}}\right]
$$

where $\left|y_{i_{j}}\right|=i_{j}$, and $\left|w_{i_{j}}\right|=i_{j}-1$.

Remark 7.4. Kuribayashi and Yamaguchi [25] compute using different methods the cohomology of free loop spaces $H^{*}(\mathcal{L} X ; \mathbb{Z} / p)$ for $X$ simply connected with mod $p$ cohomology isomorphic to $\Lambda\left(x_{i_{1}}, x_{i_{2}}\right)$, where $i_{1} \leq i_{2} \leq 2 i_{1}-2$, and $p>3$. Dualizing their result yields $H_{*}(\mathcal{L} X ; \mathbb{Z} / p) \cong \Lambda\left(y_{i_{1}}, y_{i_{2}}\right) \otimes k\left[w_{i_{1}}, w_{i_{2}}\right]$ with the generators $y_{i}$ and $w_{i}$ in the degrees indicated in the theorem above. When $p=5$ our statement applies in the same range of degrees as the Kuribayashi and Yamaguchi result. For $p>5$, though, our statement applies in a much broader range than the statement in [25], and provides an extension of their result. Note, however, that in the current work we compute $H_{*}(\mathcal{L} X ; k)$ as a $k$-module. In [25] they compute the algebra structure on $H^{*}(\mathcal{L} X ; \mathbb{Z} / p)$, in addition to the module structure. We expect that the isomorphism in Theorem 7.3 holds as an isomorphism of coalgebras, but this will be addressed in subsequent work.

As noted above, our work greatly expands the range in which we can understand the homology of the free loops on a space whose mod $p$ cohomology is exterior on two generators. We next consider spaces whose cohomology is exterior on more than two generators. When $p=2$, this was studied in work of Smith [33]. For $p>2$, while the homology of free loop spaces for spaces with exterior cohomology with one or two generators had been studied in past work, previous techniques did not easily extend beyond the case of two generators. One advantage of the new approach presented here is that it yields results in much greater generality. Indeed, using our new approach we are able to prove the following general result for spaces with exterior cohomology with $n$ generators. 
Theorem 7.5. Let $k$ be a field of characteristic $p$ and let $X$ be a simply connected space whose cohomology is exterior on a finite number of generators

$$
H^{*}(X ; k) \cong \Lambda_{k}\left(x_{i_{1}}, x_{i_{2}}, \ldots, x_{i_{n}}\right)
$$

where the $x_{i_{j}}$ are generators in odd degrees, $\left|x_{i_{j}}\right|=i_{j}$, and $i_{j+1} \geq i_{j}$. Then when $\frac{i_{n}+\sum_{j=1}^{n} i_{j}}{i_{1}-1} \leq p$, the homology of the free loop space on $X$ is given as a graded $k$-module by

$$
H_{*}(\mathcal{L} X ; k) \cong \Lambda_{k}\left(y_{i_{1}}, y_{i_{2}}, \ldots, y_{i_{n}}\right) \otimes k\left[w_{i_{1}}, w_{i_{2}}, \ldots, w_{i_{n}}\right],
$$

where $\left|y_{i_{j}}\right|=i_{j}$, and $\left|w_{i_{j}}\right|=i_{j}-1$.

Example 7.6. For appropriate choices of $p$, Theorem 7.5 recovers calculations of the homology of free loop spaces of $S U(n), S p(n), G_{2}, F_{4}, E_{6}, E_{7}$, and $E_{8}$.

In the case where the cohomology of $X$ is exterior on one generator in odd degree, our techniques yield a stronger result. We consider this case in Theorem 7.9 later in this section.

The approach to proving the above results is to use the coBökstedt spectral sequence for $\operatorname{coTHH}\left(\Sigma_{+}^{\infty} X\right)$ and exploit the additional algebraic structure developed in Section 6 for the coBökstedt spectral sequence. The following proposition will be very useful.

Proposition 7.7. Let $C$ be a cocommutative coalgebra spectrum such that $H_{*}(C ; k)$ is connected and $\operatorname{coHH}\left(H_{*}(C ; k)\right)$ is coflat over $H_{*}(C ; k)$. Then the $E_{2}$-term of the coBökstedt spectral sequence for $\operatorname{coTHH}(C)$,

$$
E_{2}^{*, *}(C)=\operatorname{coHH}_{*}\left(H_{*}(C ; k)\right),
$$



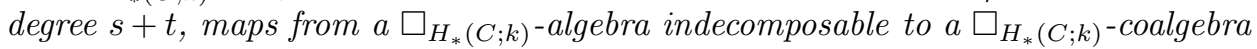
primitive.

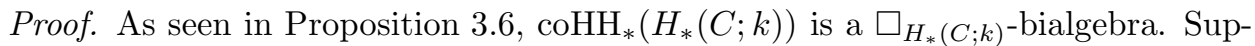
pose $d_{1}=d_{2}=\cdots=d_{r-1}=0$. We then consider what happens on $E_{r}^{*, *}(C)$. Note that $E_{r}^{*, *}(C)=E_{2}^{*, *}(C)$ is still a $\square_{H_{*}(C)}$-bialgebra, and the differential $d_{r}$ satisfies both the Leibniz rule:

$$
d_{r} \circ \mu=\mu \circ\left(d_{r} \square i d \pm i d \square d_{r}\right),
$$

and the coLeibniz rule:

$$
\triangle \circ d_{r}=\left(d_{r} \square i d \pm i d \square d_{r}\right) \circ \triangle .
$$

Here $\square$ denotes $\square_{H_{*}(C ; k)}$. Suppose $x y$ is decomposable and $d_{r}(x y) \neq 0$. Then by the Leibniz formula one of $d_{r}(x)$ or $d_{r}(y)$ is nonzero, so there is a class in lower total degree with a nonzero differential. Now consider any $w \in E_{r}^{*, *}(C)$. We can write the comultiplication on $w$ as

$$
\triangle(w)=w \square 1+1 \square w+\sum_{i} w_{i}^{(1)} \square w_{i}^{(2)} .
$$

Suppose $d_{r}(w)$ is not primitive. Then the comultiplication on $d_{r}(w)$ can be written

$$
\triangle\left(d_{r}(w)\right)=d_{r} w \square 1+1 \square d_{r} w+\sum_{i}\left(d_{r} w\right)_{i}^{(1)} \square\left(d_{r} w\right)_{i}^{(2)},
$$

where at least one of the terms $\left(d_{r} w\right)_{i}^{(1)} \square\left(d_{r} w\right)_{i}^{(2)}$ is nonzero. By the coLeibniz rule it then follows that at least one of the terms $d_{r}\left(w_{i}^{(1)}\right)$ or $d_{r}\left(w_{i}^{(2)}\right)$ must be nonzero. Therefore there is a class in lower total degree with a nonzero differential. 
We now have the pieces in place to prove Theorem 7.2.

Proof of Theorem 7.2. We consider the coBökstedt spectral sequence computing $H_{*}(\operatorname{coTHH}(C) ; k)$. This spectral sequence has $E_{2}$-term

$$
E_{2} \cong \operatorname{coHH}_{*}\left(H_{*}(C ; k)\right) \cong \operatorname{coHH}_{*}\left(\Lambda_{k}\left(y_{i_{1}}, y_{i_{2}}, \ldots, y_{i_{n}}\right)\right) .
$$

This coHochschild homology was computed in [4], Proposition 5.1, so we have

$$
E_{2} \cong \operatorname{coHH}_{*}\left(H_{*}(C ; k)\right) \cong \Lambda_{k}\left(y_{i_{1}}, y_{i_{2}}, \ldots, y_{i_{n}}\right) \otimes k\left[w_{i_{1}}, w_{i_{2}}, \ldots, w_{i_{n}}\right]
$$

where $w_{j}$ is in degree $(1, j)$. This coalgebra is coflat over the exterior algebra $\Lambda_{k}\left(y_{i_{1}}, y_{i_{2}}, \ldots, y_{i_{n}}\right) \cong H_{*}(C ; k)$, so Proposition 7.7 applies, and the shortest nonzero differential, from lowest total degree, maps from a $\square_{H_{*}(C ; k) \text {-indecomposable }}$

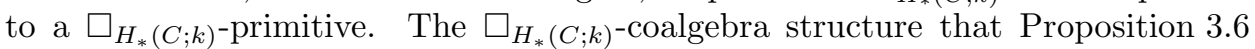
gives on the $E_{2}$-page $H_{*}(C ; k) \otimes k\left[w_{i_{1}}, w_{i_{2}}, \ldots, w_{i_{n}}\right]$ agrees with the one given in Proposition 2.18, and hence Proposition 2.18 describes the $\square_{H_{*}(C ; k)}$-primitive elements in $\operatorname{coHH}_{*}\left(H_{*}(C ; k)\right)$. However, from [4], we know that the coBökstedt spectral sequence is also a spectral sequence of $k$-coalgebras. It follows that the shortest non-zero differential, in lowest total degree, maps to a $k$-coalgebra primitive. We can see from Proposition 2.18 that there are fewer primitive elements of $\mathrm{coHH}_{*}\left(H_{*}(C ; k)\right)$ viewed as a $k$-coalgebra than viewed as a $\square_{H_{*}(C ; k)}$-coalgebra. Let $p$ denote the characteristic of $k$. Then the primitive elements of $\operatorname{coHH}_{*}\left(H_{*}(C ; k)\right)$ as a $k$-coalgebra are the elements of the form $y_{j} \otimes 1$ and $1 \otimes w_{j}^{p^{m}}$. The elements $y_{j} \otimes 1$ are in bidegree $(0, j)$ and hence cannot be hit by a differential. Similarly, the elements $1 \otimes w_{j}$ are in bidegree $(1, j)$ and cannot be hit by a differential. So the first non-zero differential, if one exists, has to hit some $1 \otimes w_{j}^{p^{m}}$, for $m \geq 1$.

We argue that for large enough values of $p$, these classes cannot be hit by a

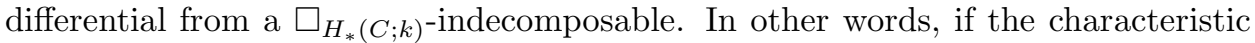
of $k$ is large enough, this spectral sequence will collapse. In Proposition 7.8 below we establish that the $\square_{H_{*}(C ; k)}$-indecomposable elements of $\operatorname{coHH}_{*}\left(H_{*}(C ; k)\right)$ are the elements of the form $x \otimes w_{i}$, where $x \in \Lambda_{k}\left(y_{i_{1}}, y_{i_{2}}, \ldots, y_{i_{n}}\right)$. Consider an indecomposable element $y_{i_{j_{1}}} \cdots y_{i_{j_{m}}} \otimes w_{i_{j}}$ and suppose $d_{r}\left(y_{i_{j_{1}}} \cdots y_{i_{m}} \otimes w_{i_{j}}\right)$ is $1 \otimes w_{i_{a}}^{p^{b}}$. Note that the bidegree of $d_{r}\left(y_{i_{1}} \cdots y_{i_{j_{m}}} \otimes w_{i_{j}}\right)$ is $\left(1+r, i_{j_{1}}+\cdots+i_{j_{m}}+i_{j}+r-1\right)$. By comparing bidegrees,

$$
1+r=p^{b} \quad \text { and } \quad i_{j_{1}}+\cdots+i_{j_{m}}+i_{j}+r-1=i_{a} p^{b} .
$$

So in particular,

$$
i_{j_{1}}+\cdots+i_{j_{m}}+i_{j}-2=\left(i_{a}-1\right) p^{b} .
$$

Since $i_{a} \neq 1$ it follows that

$$
p^{b}=\frac{i_{j_{1}}+\cdots+i_{j_{m}}+i_{j}-2}{\left(i_{a}-1\right)} .
$$

Therefore

$$
p \leq p^{b}=\frac{i_{j_{1}}+\cdots+i_{j_{m}}+i_{j}-2}{\left(i_{a}-1\right)} \leq \frac{i_{n}-2+\sum_{j=1}^{n} i_{j}}{i_{1}-1} .
$$

To complete the proof of Theorem 7.2 we prove the following lemma, which identifies the $\square_{H_{*}(C ; k)^{-}}$indecomposable elements. 
Proposition 7.8. For a field $k$, the indecomposable elements of the $\square_{\Lambda_{k}\left(y_{1}, y_{2}, \ldots y_{n}\right)^{-}}$ algebra

$$
\operatorname{coHH}_{*}\left(\Lambda_{k}\left(y_{1}, y_{2}, \ldots y_{n}\right)\right) \cong \Lambda_{k}\left(y_{1}, y_{2}, \ldots, y_{n}\right) \otimes k\left[w_{1}, w_{2}, \ldots, w_{n}\right]
$$

are of the form $x \otimes w_{i}$ for any $x \in \Lambda_{k}\left(y_{1}, y_{2}, \ldots, y_{n}\right)$.

Proof. Let $C$ denote the coalgebra $\Lambda_{k}\left(y_{1}, y_{2}, \ldots, y_{n}\right)$. Let $D$ denote the coalgebra $k\left[w_{1}, w_{2}, \ldots, w_{n}\right]$. Recall from Proposition 3.6 the $\square_{C}$-algebra structure on $\mathrm{coHH}_{*}(C) \cong C \otimes D$. In order to identify the indecomposable elements in this $\square_{C}$-algebra, we first need to identify the augmentation ideal. The augmentation

$$
\epsilon: C \otimes D \rightarrow C
$$

is given by the composite

$$
C \otimes D \stackrel{\operatorname{id} \otimes \epsilon_{D}}{\longrightarrow} C \otimes k \stackrel{\cong}{\longrightarrow} C,
$$

where $\epsilon_{D}$ is the counit for $D$. This is the map $\epsilon_{D}: k\left[w_{1}, w_{2}, \ldots, w_{n}\right] \rightarrow k$ that sends all of the $w_{i}$ to zero. Let $\bar{D}$ denote the kernel of $\epsilon_{D}$. Since we are working over a field $k$, the augmentation ideal $I(C \otimes D)$ is then given by $I(C \otimes D)=C \otimes \bar{D}$. The indecomposable elements $Q(C \otimes D)$ are determined by the exact sequence

$$
I(C \otimes D) \square_{C} I(C \otimes D) \stackrel{\mu}{\longrightarrow} I(C \otimes D) \longrightarrow Q(C \otimes D) \longrightarrow 0 .
$$

Here we view the map $\mu$ as a composite

$$
I(C \otimes D) \square_{C} I(C \otimes D) \hookrightarrow(C \otimes D) \square_{C}(C \otimes D) \stackrel{\mu_{C \otimes D}}{\longrightarrow} I(C \otimes D),
$$

where $\mu_{C \otimes D}$ is the product as in Proposition 3.6. We rewrite the exact sequence as

$$
(C \otimes \bar{D}) \square_{C}(C \otimes \bar{D}) \stackrel{\mu}{\longrightarrow} C \otimes \bar{D} \longrightarrow Q(C \otimes D) \longrightarrow 0 .
$$

Note that

$$
(C \otimes \bar{D}) \square_{C}(C \otimes \bar{D}) \cong C \otimes \bar{D} \otimes \bar{D} .
$$

This isomorphism is induced by the comultiplication followed by a twist:

$$
C \otimes \bar{D} \otimes \bar{D} \stackrel{\triangle \otimes 1 \otimes 1}{\longrightarrow} C \otimes C \otimes \bar{D} \otimes \bar{D} \stackrel{\mathrm{id} \otimes \tau \otimes \mathrm{id}}{\longrightarrow} C \otimes \bar{D} \otimes C \otimes \bar{D} .
$$

The box product $(C \otimes \bar{D}) \square_{C}(C \otimes \bar{D})$ is identified with the image of this composite inside the 4 -fold tensor product. It follows from the definition of the $\square_{C^{-}}$ multiplication in Proposition 3.6 that under this isomorphism the multiplication $\mu$ is given by

$$
\mu: C \otimes \bar{D} \otimes \bar{D} \stackrel{\operatorname{id} \otimes \mu_{D}}{\longrightarrow} C \otimes \bar{D},
$$

where $\mu_{D}$ denotes the multiplication on the classes $w_{i}^{j}$ described in Proposition 3.9. The cokernel of this map is then given by

$$
Q(C \otimes D)=C \otimes \operatorname{coker}\left(\mu_{D}\right),
$$

that is, all elements of the form $x \otimes w_{i}$.

In the case where the cohomology is exterior on only one generator, we have the stronger result that the spectral sequence always collapses, regardless of the characteristic of $k$. This result was also proven in [25] using a different approach. 
Theorem 7.9. Let $X$ be a simply connected space whose cohomology is exterior on one generator in odd degree

$$
H^{*}(X ; k) \cong \Lambda_{k}(x)
$$

Then the homology of the free loop space on $X$ is given as a graded $k$-module by

$$
H_{*}(\mathcal{L} X ; k) \cong \Lambda_{k}(y) \otimes k[w],
$$

where $|y|=|x|$, and $|w|=|x|-1$.

Proof. This follows directly from the proof of Theorem 7.2. In the case of one exterior generator in degree $i$, the formulas in the proof of Theorem 7.2 show that in order for a nonzero differential $d_{r}, r \geq 2$, to exist in the spectral sequence, the following formulas need to be satisfied:

$$
p^{b} \leq \frac{2 i-2}{i-1}=2 \quad \text { and } \quad 1+r=p^{b} .
$$

These formulas imply that $r<2$, so there is no such differential and the spectral sequence collapses.

Remark 7.10. Theorem 7.9 computes in particular the homology of free loop spaces of spheres, $H_{*}\left(\mathcal{L} S^{n} ; k\right)$, for $n>1$, odd. The homology of free loop spaces of spheres was computed classically by Ziller [36], using Morse theory, and we recover some of those results here.

Remark 7.11. A result using similar proof techniques to Theorem 7.9 is proven by Klanderman in [22]. Klanderman uses the relative coBökstedt spectral sequence to compute $\pi_{*}\left(\operatorname{coTHH}^{H k}(C)\right)$ for $C$ a cocommutative $H k$-coalgebra spectrum with $\pi_{*}(C) \cong \Lambda_{k}(y)$, for $|y|>1$, odd.

\section{REFERENCES}

[1] Marcelo Aguiar and Swapneel Mahajan. Monoidal functors, species and Hopf algebras, volume 29 of CRM Monograph Series. American Mathematical Society, Providence, RI, 2010. With forewords by Kenneth Brown and Stephen Chase and André Joyal.

[2] Vigleik Angeltveit and John Rognes. Hopf algebra structure on topological Hochschild homology. Algebr. Geom. Topol., 5:1223-1290, 2005.

[3] Haldun Özgür Bayındır and Maximilien Péroux. Spanier-Whitehead duality for topological coHochschild homology. arXiv:math.AT/2012.03966, 2020.

[4] Anna Marie Bohmann, Teena Gerhardt, Amalie Høgenhaven, Brooke Shipley, and Stephanie Ziegenhagen. Computational tools for topological coHochschild homology. Topology Appl., 235:185-213, 2018.

[5] Marcel Bökstedt. The topological Hochschild homology of $\mathbb{Z}$ and $\mathbb{Z} / p$. Unpublished.

[6] A. K. Bousfield and D. M. Kan. Homotopy limits, completions and localizations. Lecture Notes in Mathematics, Vol. 304. Springer-Verlag, Berlin-New York, 1972.

[7] A. K. Bousfield and D. M. Kan. Pairings and products in the homotopy spectral sequence. Trans. Amer. Math. Soc., 177:319-343, 1973.

[8] A. K. Bousfield and D. M. Kan. A second quadrant homotopy spectral sequence. Trans. Amer. Math. Soc., 177:305-318, 1973.

[9] Tomasz Brzezinski and Robert Wisbauer. Corings and comodules, volume 309 of London Mathematical Society Lecture Note Series. Cambridge University Press, Cambridge, 2003.

[10] D. Burghelea and Z. Fiedorowicz. Cyclic homology and algebraic $K$-theory of spaces. II. Topology, 25(3):303-317, 1986.

[11] Moira Chas and Dennis Sullivan. String topology. arXiv:math.GT/9911159, 1999.

[12] Ralph L. Cohen and John D. S. Jones. A homotopy theoretic realization of string topology. Math. Ann., 324(4):773-798, 2002. 
[13] Ralph L. Cohen, John D. S. Jones, and Jun Yan. The loop homology algebra of spheres and projective spaces. In Categorical decomposition techniques in algebraic topology (Isle of Skye, 2001), volume 215 of Progr. Math., pages 77-92. Birkhäuser, Basel, 2004.

[14] Yukio Doi. Homological coalgebra. J. Math. Soc. Japan, 33(1):31-50, 1981.

[15] Samuel Eilenberg and John C. Moore. Homology and fibrations. I. Coalgebras, cotensor product and its derived functors. Comment. Math. Helv., 40:199-236, 1966.

[16] A. D. Elmendorf, I. Kriz, M. A. Mandell, and J. P. May. Rings, modules, and algebras in stable homotopy theory, volume 47 of Mathematical Surveys and Monographs. American Mathematical Society, Providence, RI, 1997. With an appendix by M. Cole.

[17] Thomas G. Goodwillie. Cyclic homology, derivations, and the free loopspace. Topology, 24(2):187-215, 1985.

[18] Detlef Gromoll and Wolfgang Meyer. Periodic geodesics on compact riemannian manifolds. J. Differential Geometry, 3:493-510, 1969.

[19] Kathryn Hess, Paul-Eugène Parent, and Jonathan Scott. CoHochschild homology of chain coalgebras. J. Pure Appl. Algebra, 213(4):536-556, 2009.

[20] Kathryn Hess and Brooke Shipley. Invariance properties of coHochschild homology. J. Pure Appl. Algebra, 225(2):106505, 2021.

[21] A. Joyal. Quasi-categories and Kan complexes. J. Pure Appl. Algebra, 175(1-3):207-222, 2002.

[22] Sarah Klanderman. Computations of relative topological coHochschild homology. arXiv:2108.07863, 2021.

[23] Katsuhiko Kuribayashi. The Hochschild cohomology ring of the singular cochain algebra of a space. Ann. Inst. Fourier (Grenoble), 61(5):1779-1805 (2012), 2011.

[24] Katsuhiko Kuribayashi, Luc Menichi, and Takahito Naito. Behavior of the Eilenberg-Moore spectral sequence in derived string topology. Topology Appl., 164:24-44, 2014.

[25] Katsuhiko Kuribayashi and Toshihiro Yamaguchi. The cohomology algebra of certain free loop spaces. Fund. Math., 154(1):57-73, 1997.

[26] Jacob Lurie. Elliptic cohomology I. September 2016 version. Available on author's homepage.

[27] Jacob Lurie. Higher algebra. September 2017 version. Available on author's homepage.

[28] Cary Malkiewich. Cyclotomic structure in the topological Hochschild homology of DX. Algebr. Geom. Topol., 17(4):2307-2356, 2017.

[29] J. McClure, R. Schwänzl, and R. Vogt. $T H H(R) \cong R \otimes S^{1}$ for $E_{\infty}$ ring spectra. J. Pure Appl. Algebra, 121(2):137-159, 1997.

[30] Luc Menichi. The cohomology ring of free loop spaces. Homology Homotopy Appl., 3(1):193224,2001

[31] Maximilien Péroux. Rigidification of connective comodules. arXiv:2006.09398, 2020.

[32] Emily Riehl and Dominic Verity. Elements of $\infty$-category theory. Version of May 2021. Available on the first author's homepage.

[33] Larry Smith. The Eilenberg-Moore spectral sequence and the mod 2 cohomology of certain free loop spaces. Illinois J. Math., 28(3):516-522, 1984.

[34] Moss E. Sweedler. Hopf algebras. Mathematics Lecture Note Series. W. A. Benjamin, Inc., New York, 1969.

[35] Charles A. Weibel. An introduction to homological algebra, volume 38 of Cambridge Studies in Advanced Mathematics. Cambridge University Press, Cambridge, 1994.

[36] Wolfgang Ziller. The free loop space of globally symmetric spaces. Invent. Math., 41(1):1-22, 1977.

Department of Mathematics, Vanderbilt University, 1326 Stevenson Center, Nashville, TN, 37240, USA

Email address: am.bohmann@vanderbilt.edu

Department of Mathematics, Michigan State University, 619 Red Cedar Road, East LANSING, MI, 48824, USA

Email address: teena@math.msu.edu

Department of Mathematics, Statistics, and Computer Science, University of Illinois at Chicago, 508 SEO m/c 249, 851 S. Morgan Street, Chicago, IL, 60607-7045, USA

Email address: shipleyb@uic.edu 